\title{
Polymer-Assisted In Situ Synthesis of Silver Nanoparticles with Epigallocatechin Gallate (EGCG) Impregnated Wound Patch Potentiate Controlled Inflammatory Responses for Brisk Wound Healing
}

This article was published in the following Dove Press journal: International Journal of Nanomedicine

\begin{abstract}
Aditya $\mathrm{K} \mathrm{Kar}^{\mathrm{I,2}}$
Amrita Singh (iD) ${ }^{1,2}$

Nitesh Dhiman ${ }^{1,2}$

Mahaveer P Purohit ${ }^{1,2}$

Pankaj Jagdale iD $^{3}$

Mohan Kamthan (iD ${ }^{4}$

Dhirendra Singh (iD) ${ }^{3}$

Mahadeo Kumar ${ }^{3}$

Debabrata Ghosh (iD) 2,5

Satyakam Patnaik (iD) ${ }^{1,2}$

'Water Analysis Laboratory, Nanomaterial Toxicology Group, CSIR-Indian Institute of Toxicology Research (CSIR-IITR), Lucknow 22600I, Uttar Pradesh, India; ${ }^{2}$ Academy of Scientific and Innovative Research (AcSIR), CSIR-Indian Institute of Toxicology Research (CSIR-IITR), Lucknow 22600I, Uttar Pradesh, India; ${ }^{3}$ Regulatory Toxicology, CSIR-Indian Institute of Toxicology Research (CSIR-IITR), Lucknow 22600I, Uttar Pradesh, India; ${ }^{4} \mathrm{CITAR}$, CSIR-Indian Institute of Toxicology Research (CSIR-IITR), Lucknow 22600I, Uttar Pradesh, India; ${ }^{5}$ Immunotoxicology Laboratory, Food Drug and Chemical Toxicology Group, CSIRIndian Institute of Toxicology Research (CSIR-IITR), Lucknow 22600I, Uttar Pradesh, India
\end{abstract}

Correspondence: Satyakam Patnaik Water Analysis Laboratory, Nanomaterial Toxicology Group, CSIR-Indian Institute of Toxicology Research (CSIR-IITR), Vishvigyan Bhawan, 3I, Mahatma Gandhi Marg, Lucknow 22600I, Uttar Pradesh, India

Tel +9l 5222627586, Ext. 205

Fax +91 5222628227

Email satyakampatnaik@yahoo.com
Introduction: An ideal wound dressing material needs to be predisposed with desirable attributes like anti-infective effect, skin hydration balance, adequate porosity and elasticity, high mechanical strength, low wound surface adherence, and enhanced tissue regeneration capability. In this work, we have synthesized hydrogel-based wound patches having antibacterial silver nanoparticles and antioxidant epigallocatechin gallate (EGCG) and showed fast wound closure through their synergistic interaction without any inherent toxicity.

Methods and results: Wound patches were synthesized from modified guar gum polymer and assessed to determine accelerated wound healing. The modified polymer beget chemicalfree in-situ synthesis of monodispersed silver NPs $(\sim 12 \mathrm{~nm})$, an antimicrobial agent, besides lending ionic surface charges. EGCG impregnated during ionotropic gelation process amplified the efficacy of wound patches that possess apt tensile strength, porosity, and swellability for absorbing wound exudates. Further, in vitro studies endorsed them as non-cytotoxic and the post agent effect following exposure to the patch showed an unbiased response to $E$ coli $\mathrm{K} 12$ and B. subtilis. In vivo study using sub-cutaneous wounds in Wistar rats validated its accelerated healing properties when compared to a commercially available wound dressing material (skin graft; Neuskin- $\mathrm{F}^{\circledR}$ ) through better wound contraction, promoted collagen deposition and enhanced vascularization of wound region by modulating growth factors and inflammatory cytokines.

Conclusion: Synthesized wound patches showed all the desired attributes of a clinically effective dressing material and the results were validated in various in vitro and in vivo assays. Keywords: aminated guar gum, hydrogels, silver nanoparticles, epigallocatechin gallate, wound healing

\section{Introduction}

Wound is characterized as simple or austere break in structure of skin as a result of injury or severance to structural anatomy and function. ${ }^{1}$ It can extend to auxiliary tissues such as subcutaneous tissue, muscles, tendons, nerves, vessels and even to the bone, and the healing process involves complex-coordinated interactions between diverse immunological and biochemical systems. ${ }^{2}$ The whole woundhealing process proceeds in an orderly fashion, however, sometimes this cascade 
of events during the healing process breaks down due to several patho-physical conditions such as diabetes, persistent infections, poor primary treatment and other patientrelated factors. ${ }^{3}$ In case of chronic wounds, the transition from acute inflammatory phase to the subsequent proliferation and remodeling phases is impaired causing persistent inflammation marked by excessive neutrophil infiltration and its associated physiological responses like reactive oxygen species (ROS), reactive nitrogen species (RNS) and their derivatives. ${ }^{4,5}$ A delicate balance between free radicals and their scavenging systems is primordial to normal functioning of innate immunity and promote wound healing by stimulating healthy tissue. However, any insult to this equilibrium damages both the signaling and host defense capacities culminating in local and distant patho-physiological inflammatory effects. ${ }^{4,6,7}$ Delayed wound healing is a major concern, estimated to be worth $\$ 6.7$ billion worldwide and has tremendous growth potential owing to the increasing incidence of chronic wounds.

Several reports corroborate the use of efficacious antioxidants towards mitigating these enduring challenges. ${ }^{4,8-10}$ Catechins and in particular Epigallocatechin gallate (EGCG) possess ROS hunting ability and its wound-healing ability by enhancing re-epithelialization is reported. ${ }^{11,12}$ It modulates pro-inflammatory cytokines IL-1 $\beta$ and TNF- $\alpha$ and antiinflammatory cytokine, IL-10 production. $^{10}$ Besides, EGCGs' antimicrobial (both bacteriostatic and bactericidal) potency is well-studied. ${ }^{13}$

The emergence of MDR (multiple drug resistance) compels biologists to look for non-traditional antimicrobial agents. Silver, as "colloidal silver," is used as a disinfecting and microbicidal agent in various commercial products and has been used as an essential component in wound-healing products. ${ }^{14,15}$ Also, the use of silver nanoparticles (Ag NPs) in clinical parlance has seen a multifold increase in the last decade as they are equally potent to prokaryotic microorganisms and antibiotic-resistant bacterial strains. ${ }^{16-19}$

A staggering number of wound dressings are available but, choosing the right kind of dressing material to a specific type of wound is often over sighted due to lack of understanding of wound care management. Recent innovation and research in developing self-healing hydrogels resulted in their extensive use in a variety of applications, from soft robotics to tissue engineering ${ }^{20,21}$ and especially nanocomposite hydrogels are emanating as a beautiful concept to craft dressing materials with tailored properties. ${ }^{22-26}$ The synergistic interplay between the matrix and impregnated NPs results in imparting appealing features to the hydrogels like hiked mechanical strength, phase modulation, enhance hydration, microbicidal, and surface morphology in terms of charge and sterility. Additionally, the propensity to absorb, swell, and retain a large amount of water, sensitivity to $\mathrm{pH}$, temperature, or other stimuli is propitious towards wound healing. ${ }^{27-29}$ Moreover, tweaking NPs size and shape by the hydrogel's internal architecture is worth mentioning. Natural polysaccharides like Guar Gum (GG) are good immunogens, used in several wound care management and drug delivery applications. ${ }^{30-33}$ Likewise, sodium alginate (SA) a biocompatible, non-toxic, non-immunogenic, biodegradable polymer have antimicrobial property with distinct advantage of gelation in presence of divalent cations. ${ }^{34}$ Alginate's structural similarities with tissue extracellular matrices and its ability to circumvent tissue granulation were exploited in several wound care products. ${ }^{35-37}$ Gelatin, like above polysaccharides, known to impart structural integrity in terms of mechanical, chemical, and physical properties used in many pharmaceuticals and medical applications. ${ }^{35,38-40}$ Glycerol, a humectant, and a bacteriostatic agent allows the exudates to dry out and keeps hydrogel hydrated over a longer period, was also used in various wound care products. ${ }^{41}$

Therefore, the above facts substantiate our hypothesis that a composite matrix comprising all the above active components in an optimum amount will surely be beneficial towards wound-healing prospects. The present study delineates the potential of bio-inspired hydrogel nanocomposite system as an efficacious wound dressing material with many desirable critical components for quick scarfree wound healing, vis-à-vis, a commercially available collagen film dressing product, NeuSkin- $\mathrm{F}^{\circledR}$.

\section{Experimental Section}

All chemicals and reagents used in this study were of highest purity grade. Guar gum (GG), Sodium alginate (SA), Glycerol, Epigallactocatechin gallate (EGCG), Calcium chloride $\left(\mathrm{CaCl}_{2}\right)$, Silver nitrate $\left(\mathrm{AgNO}_{3}\right)$, Ethylenediamine $\left(\mathrm{C}_{2} \mathrm{H}_{8}\right.$ $\mathrm{N}_{2}$ ), Sodium borohydride $\left(\mathrm{NaBH}_{4}\right)$, 2,4,6-Trinitrobenzene Sulfonic Acid (TNBS), Minimum Essential Medium (MEM), Trypsin-EDTA, Penicillin-Streptomycin antibiotic, 3-(4,5-dimethylthiazol-2-yl)-2,5-diphenyl tetrazolium bromide (MTT), were purchased from Merck-Sigma (St. Louis, MO, USA). Gelatin (240 bloom), Nitric acid $\left(\mathrm{HNO}_{3}\right) \mathrm{AR}$ grade were sourced from SD Fine Chemicals, India. Ketamine injections after ethical clearance were purchased from the local medical store. Commercially available wound dressing material (skin graft) Neuskin- $\mathrm{F}^{\circledR}$, was obtained from Eucare Pharmaceuticals Private Limited, Chennai, India. Primary and secondary antibodies used in the present study 
were purchased from Santa Cruz, USA. MSC P5 skin keratinocytes cells were purchased from CLS cell line services (Germany). Multiplexing milliplex map kit was obtained from Merck-Millipore Corporation (USA). All other chemicals used in the study were of analytical grade.

\section{Synthesis of HG-Ag-EGCG Hydrogel Patches}

The hydrogel nanocomposites were prepared in a threestep process as described below.

\section{Step I: Synthesis of Aminated GG (AGG)}

GG was partially aminated by using prior $\operatorname{art}^{42}$ as shown in Figure 1A.

\section{Step 2: AGG-Mediated in situ Synthesis of Ag NPs (Ag@AGG)}

Stable and size-controlled Ag NPs were obtained using simple reduction chemistry where AGG plays a dual role in reducing and stabilizing agent. Conditions were optimized using critical parameters such as reaction time, $\mathrm{AgNO}_{3}$ concentration, AGG concentration and temperature of the reaction. The optimized condition, wherein $\mathrm{AgNO}_{3}$ solution $(300 \mu \mathrm{L}, 26 \mathrm{mM})$ was added to the AGG solution $(5 \mathrm{~mL}, 1 \%$ $\mathrm{w} / \mathrm{v}$ ) was chosen for the synthesis of Ag NPs. Post $30 \mathrm{~min}$, the NPs formation was observed by visual color change followed by UV-Vis spectroscopic characterization.

\section{Step 3: Preparation of Hydrogel-Ag NPs Patch (HG-Ag) and hydrogel-Ag-EGCG (HG-Ag-EGCG) Patch}

Initially, $1 \%(\mathrm{w} / \mathrm{v})$ stock solutions of AGG, SA, and gelatin were prepared. Bare hydrogel (HG) synthesis was achieved by thoroughly mixing equal amount of AGG, SA, and gelatin solutions $(1: 1: 1, \mathrm{v} / \mathrm{v})$ followed by the addition of glycerol $(0.5 \% \mathrm{v} / \mathrm{v})$ and $\mathrm{CaCl}_{2}(2.5 \% \mathrm{w} / \mathrm{v})$. The solution mixture was homogenized, casted in 6-well plate and kept at $-20^{\circ} \mathrm{C}$ overnight, followed by lyophilization for 2 days $\left(-105^{\circ} \mathrm{C}\right)$ to obtain disc-shaped dehydrated hydrogels. Hydrogel formation occurred due to coordinative interaction between AGG,

A

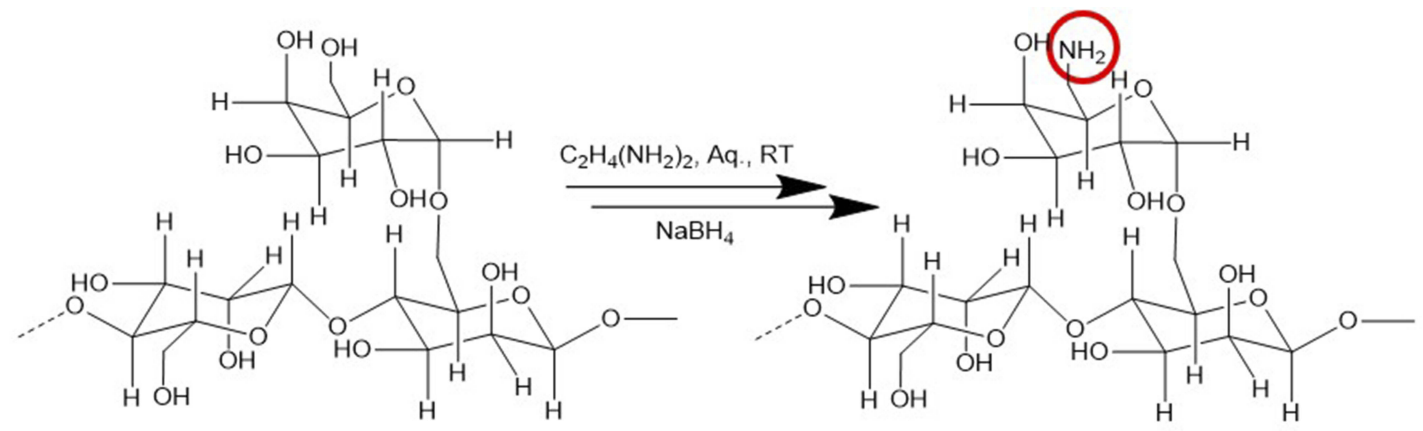

\section{Guar gum (GG)}

B

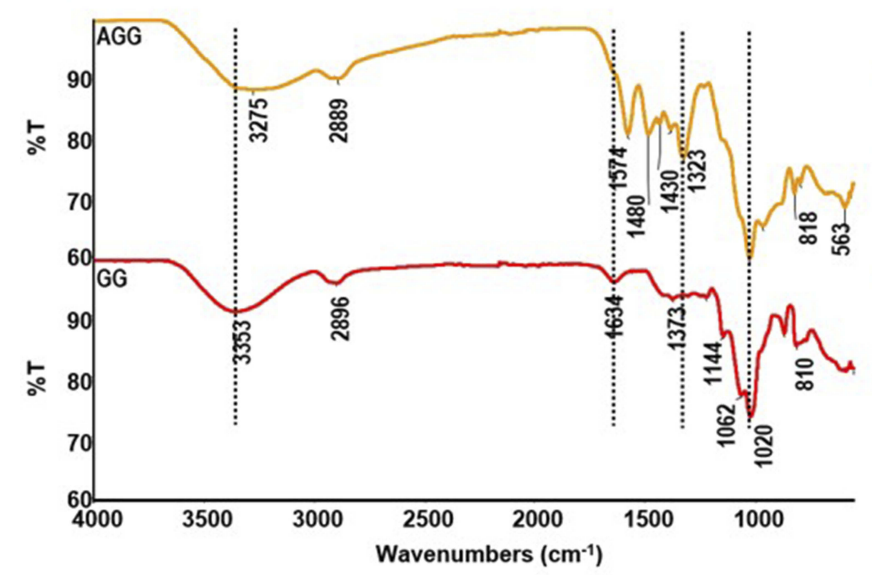

Aminated guar gum (AGG)

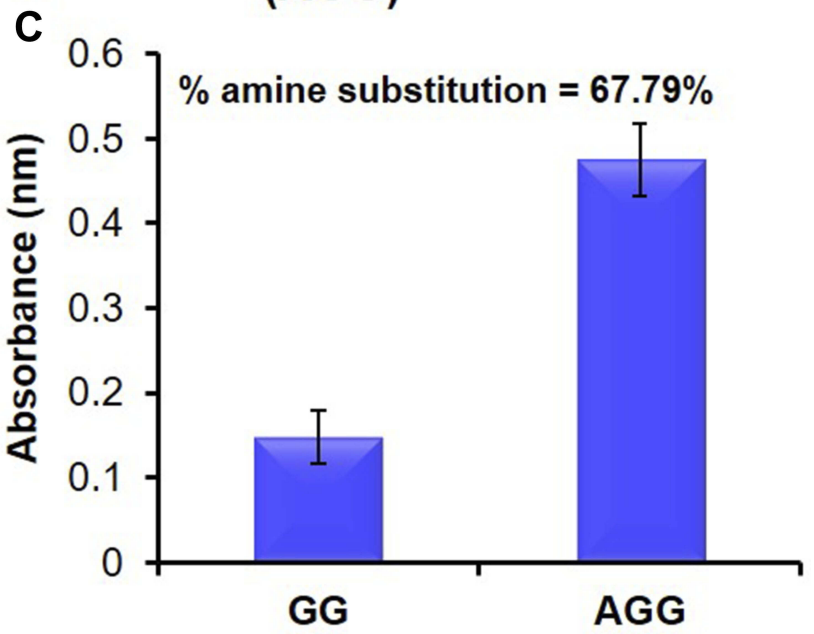

Figure I Modification of natural polymer guar gum (GG). (A) Synthetic scheme of amination of GG. (B) FTIR spectra showing the successful modification of GG to aminated guar gum (AGG). (C) Degree of substitution in the polymer backbone by TNBS assay. 
calcium ions (cationic) and SA (anionic). Similarly, HGEGCG, HG-Ag, and HG-Ag-EGCG patches are prepared following the above protocol except that $50 \mu \mathrm{g} / \mathrm{mL}$ of EGCG for HG-EGCG, Ag@AGG instead of AGG for HG-Ag, and both, EGCG and Ag@AGG for HG-Ag-EGCG, were taken in the final homogenized solution prior to lyophilization.

\section{Hydrogel Characterizations}

Structural changes from GG to AGG were recorded by ATR FT-IR spectra on a single beam Nicolet ${ }^{\mathrm{TM}}$ iSTM5 FT-IR Spectrometer. Surface morphology and qualitative elemental analysis of all the hydrogel nanocomposites were investigated by SEM coupled with EDX (SEM, Quanta FEG 450, FEI, and Netherland). Actual size and shape of Ag NPs were obtained by TEM analysis on FEI TechnaiG-2 spirit, Netherland operated at $80 \mathrm{kV}$ with $15000 \times$ magnifications. The sample crystallinity was examined using multipurpose X-ray diffraction system with built-in intelligent guidance from Rigaku (SmartLab SE) with a computer-controlled SmartLab Studio II software. X-ray diffraction (XRD) measurements of synthesized silver nanoparticles cast onto glass slides were done on the above diffractometer operating at a voltage of $60 \mathrm{kV}$ and current of $20 \mathrm{~mA}$ with $\mathrm{Cu} \mathrm{K}(\alpha)$ radiation of $1.54187 \mathrm{~nm}$ wavelength. The scanning as done in the region of $2 \mathrm{~h}$ from 20 to 80 at $0.02 / \mathrm{min}$ and the time constant was $2 \mathrm{~s}$. UV-Vis double beam spectrophotometer (Perkin Elmer, lambda 35, USA) was used for colorimetric assessment of synthesized Ag NPs. Hydrodynamic radius and zeta potential of Ag NPs were calculated by Dynamic Light Scattering (DLS) approach using Zetasizer Nano-ZSP Malvern, UK equipped with HeNe laser $(633 \mathrm{~nm})$. For the rheological measurements, $0.5 \mathrm{~g}$ of hydrogels was used. Raman spectra of HG-Ag-EGCG were measured using a WITEC Alpha 300AR (Germany) laser with a wavelength of $532 \mathrm{~nm}$. Calibration was initially performed using an internal silicon reference at $520 \mathrm{~cm}^{-1}$. The spectra were measured from 500 to $3800 \mathrm{~cm}^{-1}$. All samples were deposited onto glass slides in powdered form without any solvent. The rheological properties were studied with the Anton Paar Modular Compact Rheometer equipment (MCR 102 parallel-plate system, radius $=25 \mathrm{~mm}$ ) at $25^{\circ} \mathrm{C}$. A shear rate $\gamma=100 \mathrm{~s}^{-1}$ was applied for $10 \mathrm{~s}$.

\section{Quantification of Amine Groups on AGG}

The percentage substitution of the amino group in AGG was determined as per prior art. ${ }^{42}$ Briefly, samples of GG and AGG (5 mg each) were dissolved (1 mL) in Milli-Q. To this solutions, $1 \mathrm{~mL}$ of $\mathrm{NaHCO}_{3}(4 \% \mathrm{w} / \mathrm{v})$ and $1 \mathrm{~mL}$ of freshly prepared TNBS solution $(0.5 \%, \mathrm{v} / \mathrm{v})$ were added and kept at $60^{\circ} \mathrm{C}$ for $4 \mathrm{hrs}$. Subsequently, $1 \mathrm{~mL}$ each of the reacted solution was transferred to fresh test tubes containing $\mathrm{HCl}(3 \mathrm{~mL}, 6 \mathrm{M})$. After incubating at $40^{\circ} \mathrm{C}$ for $1.5 \mathrm{hrs}$, absorbance of the formed trinitrophenyl complex was recorded at $420 \mathrm{~nm}$. The percentage of amino groups substituted in the GG polymeric backbone was calculated using Equation (1) written below,

$$
\mathrm{A}=\frac{\mathrm{Ab}-\mathrm{Gb}}{\mathrm{Ab}} \times 100
$$

where in $\mathrm{A}=$ percentage amine substitution $\%, \mathrm{Ab}=$ absorbance of $\mathrm{AGG}$ and $\mathrm{Gb}=$ absorbance of $\mathrm{GG}$.

\section{Porosity of Hydrogel Wound Patch}

The porosity of the prepared HG, HG-Ag, HG-EGCG and HG-Ag-EGCG hydrogel nanocomposites was determined using prior art. ${ }^{43}$ Briefly, the samples were immersed in absolute ethanol until saturation. The weights of sample prior and post-immersion were used to obtain the porosity following Equation (2), where W1 and W2 are weights prior and postimmersion in alcohol, respectively. V1 is the volume of alcohol before immersion and $\rho$ is a constant (the density of alcohol). Experiments were performed in triplicates.

$$
\mathrm{P}=\frac{\mathrm{W} 2-\mathrm{W} 1}{\rho \mathrm{V} 1} \times 100
$$

\section{Swelling Ratio of Hydrogel Nanocomposites}

Swelling kinetics of HG, HG-Ag, HG-EGCG, and HG-Ag -EGCG hydrogel nanocomposites was determined by weighing swollen hydrogel samples at pre-determined time points. Surface adhered water molecules were removed by gently tapping with a blotting filter paper followed by immediate weighing. The degree of swelling is calculated from Equation (3).

$$
\mathrm{DS}=\frac{\mathrm{Ww}-\mathrm{Wd}}{\mathrm{Wd}} \times 100
$$

where in DS is the degree of swelling; Ww and $\mathrm{Wd}$ represent the wet and dry weight of the sample, respectively.

\section{Release Profile of EGCG and Ag NPs}

EGCG release study was performed both in phosphatebuffered saline (PBS; pH 7.4) and DMEM incomplete medium over 15 days, mimicking physiological $\mathrm{pH}$ and wound bed condition, respectively. HG-Ag-EGCG (5 mg) was suspended in PBS ( $2 \mathrm{~mL}, 10 \mathrm{mM}, \mathrm{pH} 7.4)$ and transferred to a dialysis bag (12kD MWCO) suspended in screw cap glass 
bottle containing PBS (20 mL) as dialysis medium. However, it is to be kept in mind that release profile of drug gets hampered when MWCO of dialysis cassettes was not chosen appropriately. ${ }^{44}$ The bottle was shaken gently at $37^{\circ} \mathrm{C}$ in an incubator shaker, and at predetermined time intervals, aliquots were drawn, subjected to UV analysis followed by replenishment with fresh buffer. A pre-drawn calibration curve of EGCG was used to calculate EGCG release. Similarly, release profile of Ag NPs and EGCG from HG-Ag-EGCG in DMEM medium kept in six-well culture plates containing $10 \mathrm{~mL}$ of DMEM incomplete medium and incubated in a $\mathrm{CO}_{2}$ incubator at $37^{\circ} \mathrm{C}$ was performed. This media was further used for Ag quantification by AAS and for cell viability assay.

\section{Ag NP Quantification}

A standard curve was plotted in the range of 0.25 to $5 \mathrm{mg} / \mathrm{L}$, prepared by diluting NIST standard solution $(1000 \mathrm{mg} / \mathrm{L})$. The media was collected from day 1 to day 15, digested with $\mathrm{HNO}_{3}$ and subsequently analyzed by Atomic Absorption Spectrophotometer (ZEEnit 700, Analytik Jena AG, Germany) equipped with high-intensity hollow cathode lamp of Ag. WinAAS software was used for data integration and processing. Finally, Ag concentration in samples was estimated using the above standard curve.

\section{Sterilization of Hydrogel Wound Patches}

Synthesized hydrogel wound patches were kept for UV sterilization in cell culture hood for 30 mins to remove any contamination before the application.

\section{In vitro Cell Viability Assay}

The in vitro cytotoxicity of HG-Ag-EGCG was evaluated using our previous published literature. ${ }^{33}$ Briefly, 96 well cell culture plates were used containing 10,000 cells per well and incubated overnight in $\mathrm{CO}_{2}$ incubatorafterwards they were treated with timely aspirated media of HG-Ag-EGCG for $24 \mathrm{hrs}$, followed by $10 \mu \mathrm{L}$ treatment of MTT for another 4 hrs. DMSO $(100 \mu \mathrm{L} /$ well) was used to dissolve the formazan crystal and absorbance was read at $570 \mathrm{~nm}$ on a microplate reader ( $\Omega$ fluostar, BMG labtech). The cell viability was expressed as the percentage of live cells relative to the control. All experiments were performed in triplicate.

\section{Antibacterial Potency of Various Hydrogel Patches}

To discern the antibacterial efficacy of hydrogel patches, zone inhibition and growth curve methodology were used.
Four kinds of strains (both Gram +ve and Gram -ve), E. coli K12 (MTCC-1302), Pseudomonas aeruginosa (MTCC-424), B. Subtilis (MTCC-441), and Staphylococcus aureus (lab strain) were inoculated in Luria Bertani (LB) medium and cultured at $37^{\circ} \mathrm{C}$ for $12 \mathrm{hrs} .100 \mu \mathrm{L}$ of each of these cultures were spread on different LB plates. Each of the synthesized hydrogel patches were placed on the bacterial plates and left for incubation overnight. HG was used as control. Presence of halo indicates the antibacterial character of wound patch. Similarly, in growth inhibition study, 0.6 OD of each bacterial strain culture was taken followed by inoculation in $5 \mathrm{~mL}$ LB broth containing a fixed weight of various hydrogel patches. The growth of the cultures was assessed at predefined time points.

\section{In vivo Wound-Healing Efficacy of Hydrogel Patches}

The wound-healing characteristics of the patches were evaluated using Wistar rat model. All experiments were performed with the approval of the Institute's Animal Ethics Committee bearing registration number and date of registration as 54/GO/RBi/S/99/CPCSEA dated 28.03.2017. The guidelines followed from The Committee for the Purpose of Control and Supervision of Experiments on Animals (CPCSEA), Ministry of Fisheries, Animal Husbandry \& Dairying, Department of Animal Husbandry, Dairying \& Fisheries, Govt. of India on laboratory animal use. Wistar rats (180-220 $\mathrm{g}$ and 3-5 weeks of age) were used in this study. Animals were acclimatized for 7 days prior to the start of experiments. The rats were randomly divided into six different groups (HG-Ag-EGCG, HG-Ag, HG-EGCG, $\mathrm{HG}$, commercial NeuSkin- $\mathrm{F}^{\circledR}$ and control untreated) containing three animals $(n=3)$ each. Animals were allowed to take normal rat feed and water ad libitum. Rats were anesthetized by intramuscular injection of Ketamine $(60.0 \mathrm{mg} / \mathrm{kg})$ prior to wounding followed by depilation of wound area and cleaning with alcohol. Full-thickness wounds of $10 \mathrm{~mm}^{2}$ were created with surgical scissors and forceps. The wounds were immediately covered by a particular hydrogel patch as per the above groups. NeuSkin- $F^{\circledR}$ group was used as positive control while rats of bare wound group served as a negative control. Every rat was housed individually in a cage under normal room temperature. The wounds were examined and photographed at pre-determined time points to estimate the reduction of their size. Animals were sacrificed on designated days (3, 6, 9, 12 and 15 days) and at each time point, the skin wound 
tissue of the rat was excised, fixed in $10 \%$ formalin and wound size measured at the time of surgery and at the time of biopsy were used to calculate the percent reduction in wound size:

$$
\text { Wound size reduction }(\%)=\frac{\mathrm{a} 1-\mathrm{a} 2}{\mathrm{a} 1} \times 100
$$

where a1 and a 2 are the initial and final wound area, respectively.

\section{Histopathological Analysis and Immunofluorescent Staining}

The removed skin specimens from each group collected on designated time points were fixed in $10 \%$ buffered formalin, processed, embedded in paraffin, and then sectioned perpendicular to the wound surface into $5 \mu \mathrm{m}$ sections by standard procedures. Sections were stained with hematoxylin-eosin (H\&E), Masson trichrome and Immunohistochemical reagent; KI 67 (1:100, Abcam). For immunohistochemistry, de-paraffinized sections were incubated overnight at $4^{\circ} \mathrm{C}$ with primary antibody (1:500 in blocking buffer). Sections were washed thrice with the PBS (1X) for 15 min each followed by incubation with appropriate secondary antibodies for 2 hrs. Nuclei were stained with DAPI $(1 \mu \mathrm{g} / \mathrm{mL})$ for 2 min, washed and sections were mounted on glass slides using the antifade mounting medium (Invitrogen). Slides were observed under confocal microscopy in 20x magnification (Zeiss LSM 880).

\section{Biochemical Analysis and Toxicity Assessment of Different Hydrogel Patches}

To assess any insult or toxicity to the hepatic and renal system possibly from different wound patches, blood samples were collected for each group at the time of necropsy towards biochemical analysis. Animals were anesthetized with Ketamine, and blood samples were collected in vacutainer via the cardiac puncture through a syringe attached to 22 gauge needle. The urea, creatinine, alanine transaminase (ALT) and aspartate transaminase (AST) were estimated using automated clinical chemistry analyzer (Rx Daytona, Randox, U.K.).

\section{Detection of Cytokine Level in Serum}

Blood samples collected through cardiac puncture of the rats were allowed to clot at ambient temperature for about $60 \mathrm{~min}$. Samples were centrifuged at $2000 \mathrm{~g}$ for $10 \mathrm{~min}$ at $4^{\circ} \mathrm{C}$ for serum collection. Serum samples from respective groups were measured for inflammatory cytokine (IL-6, IL-10, IFN- $\gamma$, and TNF- $\alpha$ ) and vascular endothelial growth factor (VEGF) plated in triplicate using a Milliplex rat cytokine assay kit (Millipore) in Bio-Plex MAGPIX multiplex reader (Bio-Rad). Fluorescence associated with different antibody-coated magnetic beads was read to quantify respective cytokines.

\section{Statistical Analysis}

Statistical analysis was performed by GraphPad Prism (Graphpad Software Inc., San Diego, CA). All data are presented as the mean \pm standard deviation (SD), and a $p$-value of less than 0.05 was considered statistically significant. The difference between the two groups was analyzed by unpaired, two-tailed Student's $t$-test. Similarly, one-way ANOVA multiple comparisons with Tukey's test were applied to analyze the differences between multiple groups.

\section{Results and Discussion Synthesis and Characterization of Hydrogel Patches}

GG was aminated to AGG to ensure reagent-free green reduction of $\mathrm{AgNO}_{3}$ into $\mathrm{Ag} \mathrm{NPs}$, as shown in Figure 1A. The ATR-FTIR analysis of GG and AGG showed in Figure 1B matches with data reported earlier. ${ }^{45}$ Moreover, GG and AGG also show the glycosidic (C-O-C) linkage of the pyranose ring at $1020,1144 \mathrm{~cm}^{-1}$ and primary alcohol C-O stretching vibrations corresponding to GG moiety at $1141 \mathrm{~cm}^{-1}$. Detailed characterization of various wound patches was assessed by FT-IR, XRD and Raman spectroscopy are discussed and presented in Figures $\mathrm{S} 1$ and $\underline{\mathrm{S} 2}$, respectively.

Further, the percentage of amine modification quantified by TNBS assay showed the degree of substitution of amino groups to be $67.79 \%$, as shown in Figure $1 \mathrm{C}$ and agrees with the literature. ${ }^{42}$ One of the aims of the present study is facile green synthesis of Ag NPs in a reagent-free condition, and therefore, $-\mathrm{NH}_{2}$ moieties were introduced in the polymeric backbone of GG yielding AGG. After several iterations, with the optimized condition of $1 \% \mathrm{w} / \mathrm{v}$, AGG solution can easily reduce $26 \mathrm{mM} \mathrm{AgNO}_{3}$ into homogenous yellow colloidal solution Ag NPs (Figure 2A). The UV spectrum shown in Figure 2B confirms the synthesis of AGG capped Ag NPs (Ag@AGG). The TEM image and size distribution histogram depicted in Figure $2 \mathrm{C}$ and $\mathrm{D}$ displayed monodisperse Ag NPs in range of 6 to $12 \mathrm{~nm}$. 
A

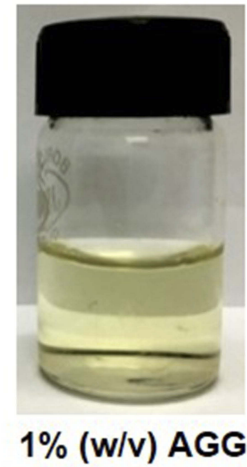

C

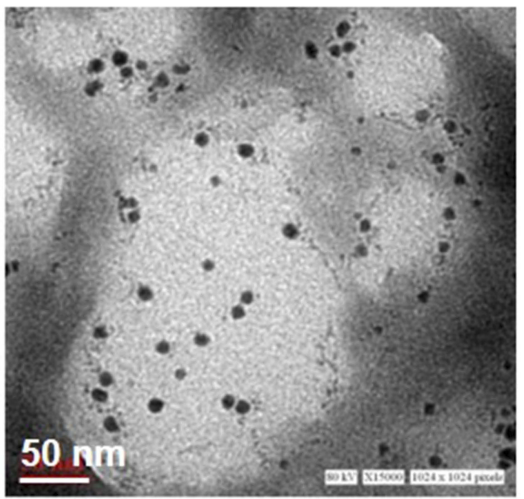

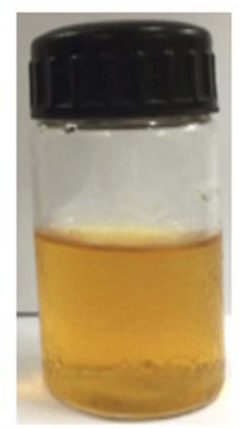

Ag@AGG NPs

D

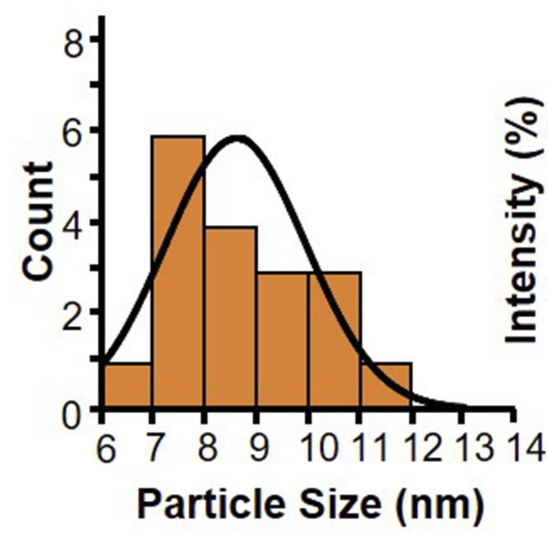

B
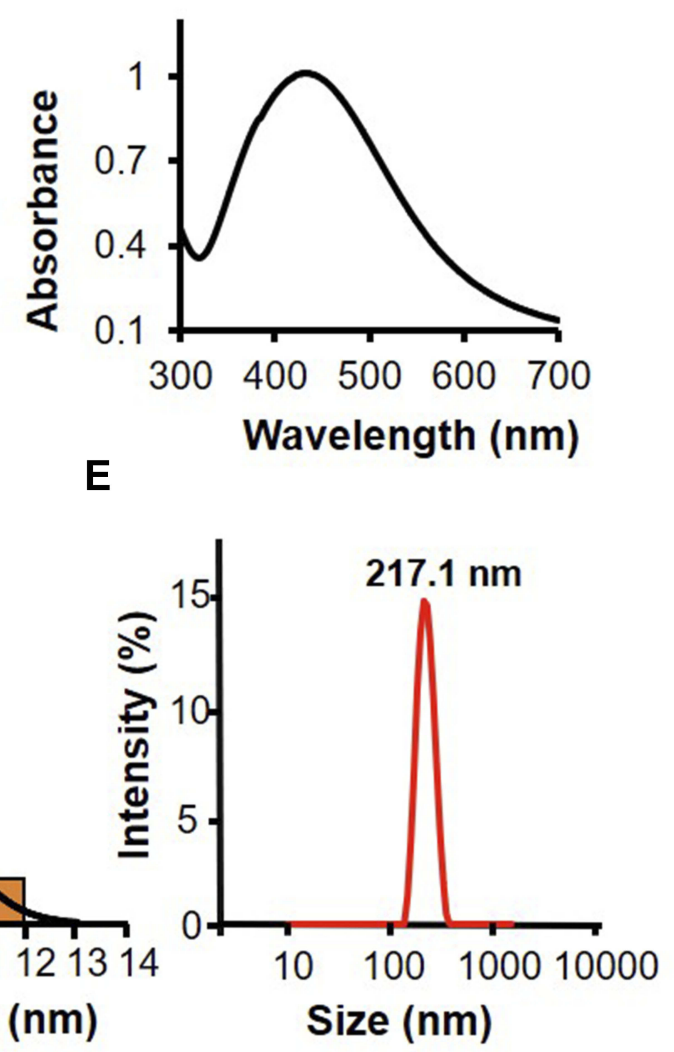

Figure 2 Characterization of AGG-assisted Ag NPs (Ag@AGG NPs). (A) Representative scheme of Ag@AGG NPs synthesis. (B) Characteristic UV peak of Ag NPs at 420 nm. (C and D) Representative TEM photomicrograph of Ag@AGG NPs reveals the size in the range of 6-12nm (scale bar=50nm) and size distribution of Ag@AGG NPs by ImageJ software, respectively. Photomicrographs indicates a successful synthesis of Ag NPs having a spherical shape in nature. (E) DLS size distribution showing Ag@AGG NPs average diameter $(217.1 \pm 10 \mathrm{~nm})$.

Figure 2E shows the hydrodynamic size of Ag@AGG NPs determined by DLS found to be $217 \pm 10 \mathrm{~nm}$ with polydispersity (PDI) of 0.197 which support the larger diameter after the hydration. The aliphatic $\mathrm{O}-\mathrm{H}$ and $-\mathrm{NH}_{2}$ groups are prone to form intermolecular hydrogen bonds which enhance the radius of NPs. The zeta potential analysis has revealed slightly negative surface charges ranging from -2.6 to $-1.59 \mathrm{mV}$. Figure 3A (upper panel) depicts the digital images of various synthesized hydrogel patches HG, HG-Ag, HG-EGCG, and HG-Ag-EGCG with welldefined fluffy and spongy structure with or without active components. Visually, the color intensity successively enhanced due to the presence of Ag NPs, EGCG, and both together in hydrogel patches.

Further, the SEM images (Figure 3F) reveal about the microcapillary channels formed due to the interconnection of pores which remain unaffected even after incorporation of active components, observed at higher magnification (70X). Upon probing further, the presence of Ag NPs can be seen embedded in the hydrogel surface as shiny, granular structures (inset image, yellow arrows in the second image of Figure 3F). The semi-qualitative estimation of $\mathrm{Ag}$ content in HG-Ag-EGCG was determined by EDX, as shown in Figure 3B. Presence of C, O, Na and Ca appeared due to AGG and SA. Calcium is known to chelate SA and is used, therefore, as a binder to provide mechanical strength to HG-Ag-EGCG matrix. All the hydrogel patches showed a rapid and higher degree of saturated swelling in the range from $1400 \%$ to $1800 \%$ in Milli Q within 10 min Figure 3C. The higher swelling phenomena are due to the formation of interconnected pores and microchannels that offers an avenue for higher water absorption rate in the hydrogels. ${ }^{46}$ The high swelling ratios prompted us to look for the intricate structure inside the formulations. The porosity of different hydrogel patches was, therefore, evaluated using an alcohol displacement method. ${ }^{43}$ Interestingly, the observed porosity of HG, HG-Ag, HG-EGCG, and HG-Ag-EGCG remains at $53 \%$ to $56 \%$ or unaltered, even after the incorporation of EGCG and Ag NPs shown in (Figure 3D). 
A

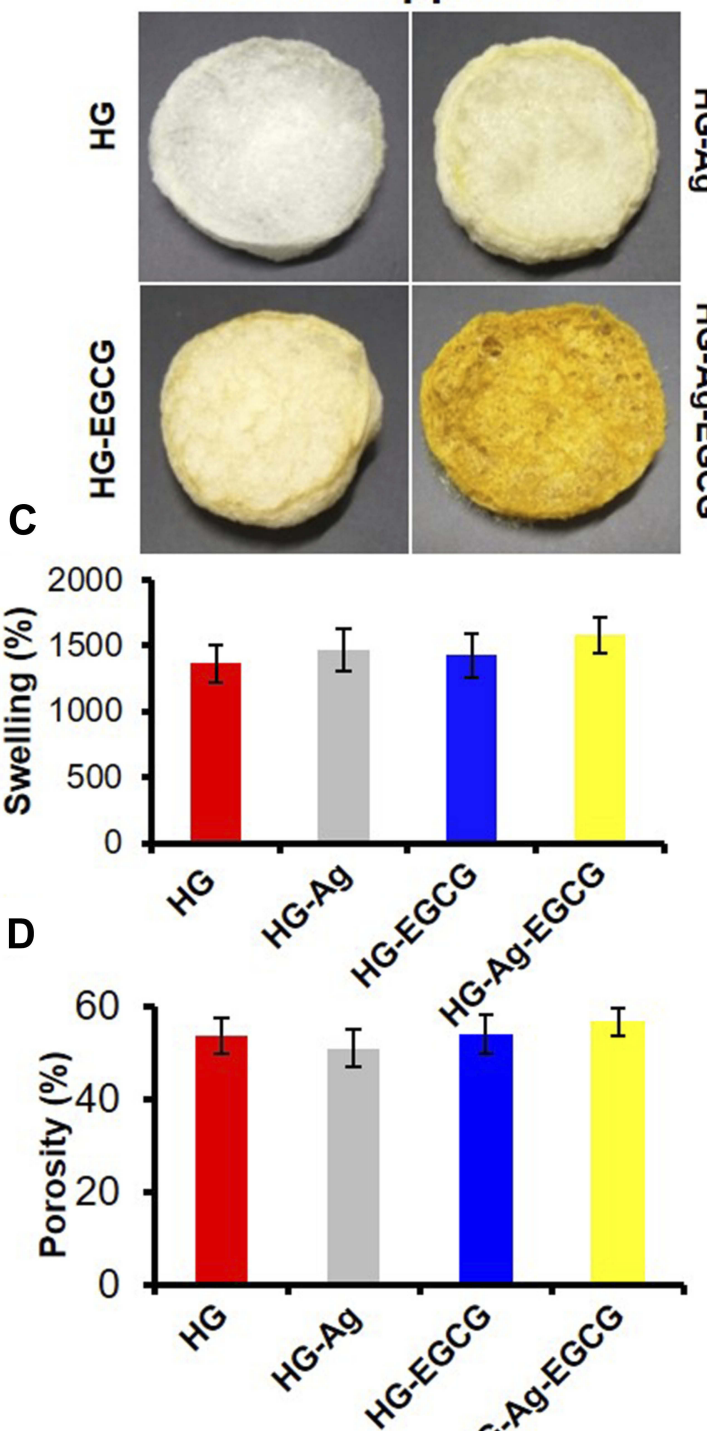

$\mathbf{F}$

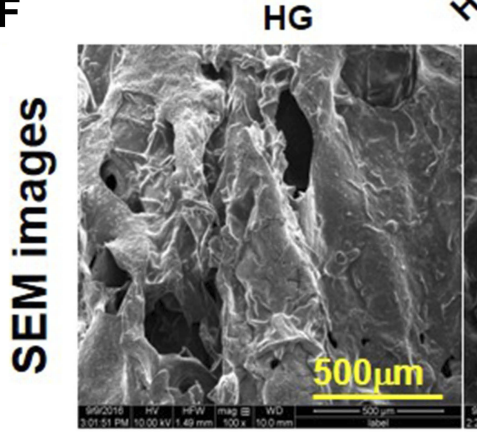

B
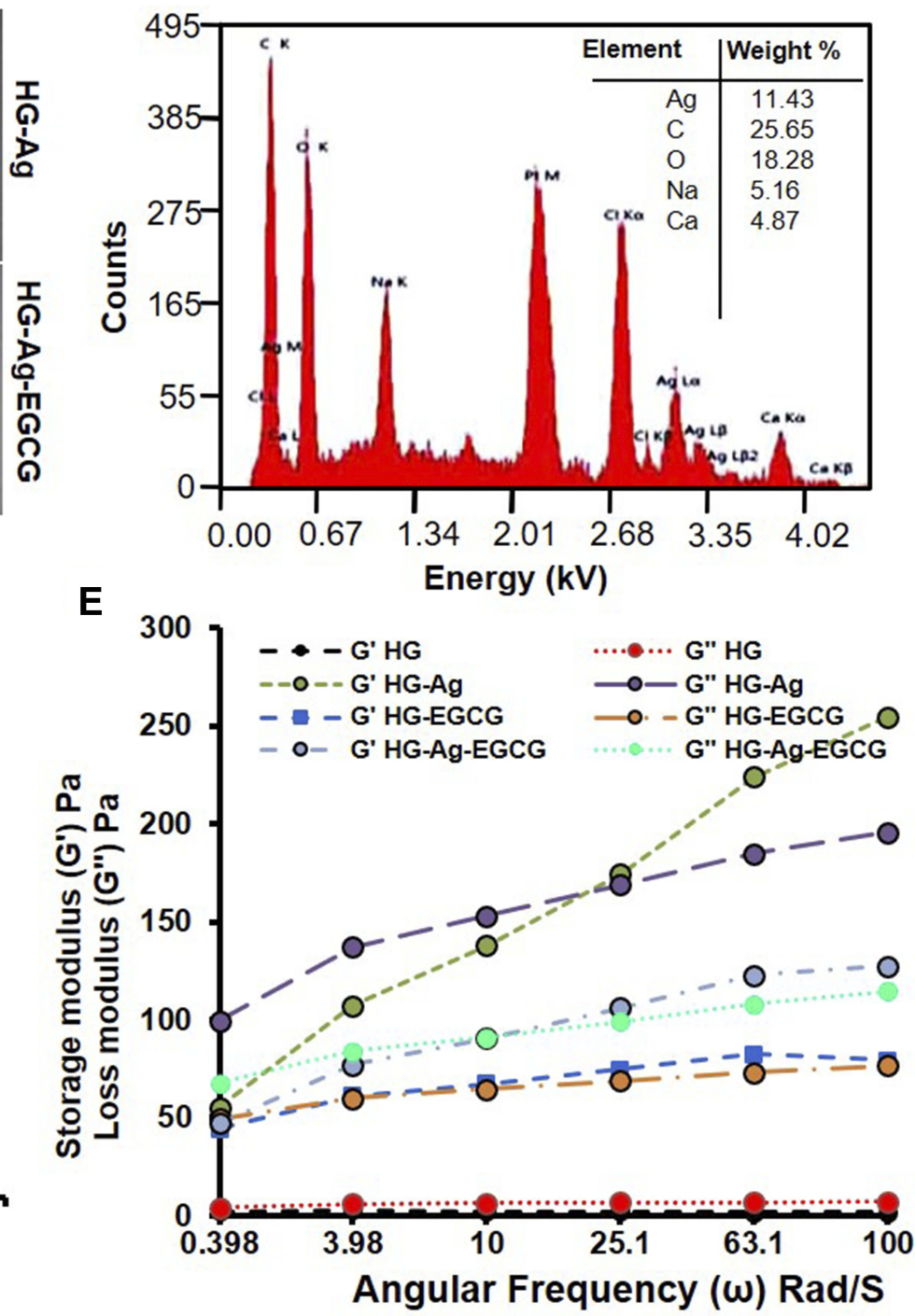

HG-EGCG

HG-Ag-EGCG

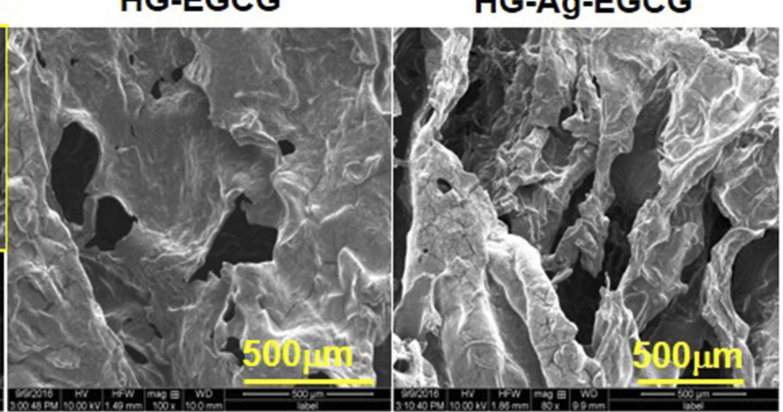

Figure 3 Synthesis and characterization of hydrogels and hydrogel patches. (A) Digital image of the various formulation showing the distinct color change with subsequent incorporation of the active materials. (B) EDAX analysis shows the chemical composition of HG-Ag-EGCG and Ag concentration in HG-Ag-EGCG was recorded to be II.43\%. (C) Swelling behavior of different hydrogel formulations. (D) Porosity of different hydrogel patches. (E) Young's modulus reveals sufficient mechanical strength of hydrogel formulations concerning storage and loss modulus (mean $\pm \mathrm{SD}, \mathrm{n}=3$ ). Scale bar $=500 \mu \mathrm{m}$ except for HG-Ag where it is $50 \mu \mathrm{m}$. $(\mathbf{F}) \mathrm{SEM}$ photomicrographs show the flexible porous networks of different formulations. Ag NPs formation in HG-Ag hydrogel patch (Yellow arrows showing the Ag NPs in the inset image).

We postulate the moderate porosity of the hydrogels would be beneficial to absorb wound exudates optimally without drying the wound surface as porosity is often pivotal in enhancing the distribution of nutrients. ${ }^{47}$ Thus, dynamic rheology analysis of HG, HG-Ag, HG-EGCG, and HG-AgEGCG was done to assess the mechanical strength of 
successive modification. The test involves measuring the modulus at a constant temperature with frequency sweep $(0-100 \mathrm{rad} / \mathrm{s})$. Figure $3 \mathrm{E}$ shows the loss modulus (G') of $\mathrm{HG}$ to be higher than the storage modulus $\left(G^{\prime}\right)$ reflecting high strain amplitude and fluid-like structure, while other formulations showed reciprocal relation due to change in the interfacial properties of gel and referred as elastic solid. ${ }^{48,49}$ Understandably, G' value of HG-Ag was maximum due to induced crystallinity in the polymeric matrix after the in-situ incorporation of Ag NPs which subsequently reduced post-EGCG encapsulation in HG-Ag-EGCG. Intramolecular hydrogen bonding was also introduced after the encapsulation of EGCG in hydrogel that facilitated selfsupporting ability in $\mathrm{HG}$ as compared to native HG. The above facts point mechanical strength was incremental in order of $\mathrm{HG}<\mathrm{HG}-\mathrm{EGCG}<\mathrm{HG}-\mathrm{Ag}-\mathrm{EGCG}<\mathrm{HG}-\mathrm{Ag}$. The relative material viscosity of $\mathrm{HG}, \mathrm{HG}-\mathrm{Ag}, \mathrm{HG}-\mathrm{EGCG}$ and HG-Ag-EGCG hydrogel patches was observed to be 1030 , 24,983, 12,429 and 16,958 $\mathrm{Pa}$, respectively, also corroborates the above assumption. Moreover, the mechanical flexibility of the patch in terms of the twist and turn is shown in the supplementary Figure S3, wherein the patch regains its original form when released from the bending position.

\section{Cellular Compatibility and Ag NPs/EGCG Release from HG-Ag-EGCG}

An ideal wound patch favors the optimal unloose of its active components at the wound bed towards rapid wound closure. To gauge the efficacies of all the active components of HG-Ag -EGCG vis-à-vis their cooperativity with antimicrobial and biocompatibility traits, release studies were assessed over 15 days. Prior works suggest Ag NPs with an average size in the range of $20-45 \mathrm{~nm}$ could effectively inhibit the bacterial population of several strains at MIC of $2-4 \mathrm{ppm}^{16}$ In our case, we observed a cumulative release of $\sim 4.1 \mathrm{ppm}$ of silver over 15 days, as shown in Figure 4A suggesting an optimal antibacterial environment at the wound site. Interestingly, there is almost 2-2.5 ppm initial burst release of Ag NPs at the end of the 24hrs, followed by a sustained release. Additionally, mimicking the wound site condition, EGCG release was checked in the culture medium for 15 days, and we found on day 1 , the release was $18.6 \%$ which increased to $59.8 \%$ on day 2 , and finally reached $79.8 \%$ on day 8 . Post 8 days it remains more or less constant indicating a controlled and slow release profile (Figure 4B).

The cell viability data of normal Murine Skin Keratinocyte cell lines, MSC-P5, show good biocompatibility attribute of
HG-Ag-EGCG compared to control. About $75-80 \%$ of cells remained viable over all the time points till day 15 as shown in Figure 4C. We presume, the plausible reason for this biocompatibility might be because of the controlled release of biochemical modulators (Ag NPs and EGCG) from the polymer matrix, as it is well known that higher concentration of $\mathrm{Ag}$ is toxic to the skin. ${ }^{50}$ Figure 4D shows EGCG diffusion kinetics in PBS (pH 7.4) and we postulate, HG-Ag-EGCG will likely ensure a sustained release in vivo with initial bolus dose that might be required to suppress preliminary influx of inflammatory molecules for the subsequent transition to proliferation and remodeling phase. It is well reported that the inflammatory phase lasts somewhere between a few minutes after the injury to about 3 days, post to which the production of proinflammatory molecules should recede. ${ }^{1}$ The incorporation of EGCG in our system will accentuate this repair process by allowing a smooth transition to the proliferative and remodeling phases with re-establishing of the redox homeostasis by scavenging excess ROS and RNS molecules. Collectively the above observations endorse HG-Ag-EGCG to be cytocompatible with a propensity towards antimicrobial and antioxidant property resulting in faster wound healing.

\section{Antibacterial Potency of $\mathrm{Hg}-\mathrm{Ag}$-EGCG Nanocomposite Against Bacterial Strains}

As Ag NPs are a well-known antibacterial agent, we next determined whether HG-Ag-EGCG also retains the same efficacies. We tested our wound patch against gram-positive (B. subtilis, S. aureus) and gram-negative bacteria (E. coli, $P$. aeruginosa) both by zone inhibition and growth curve analysis (Figure 5). As anticipated, $\mathrm{HG}$ formulation without Ag NPs and EGCG showed negligible inhibitory effects in both Gram +ve and Gram -ve strains (zone A), whereas, HGAg, HG-EGCG, and HG-Ag-EGCG hydrogel nanocomposites showed substantial inhibitory potency (zone B, C, D). A higher concentration of EGCG (50 ppm) in the final formulation did not augment the inhibitory effect. The sustained and controlled release of Ag NPs as discussed in the above section indeed offers a unique advantage in the sense that our hydrogel nanocomposites exert antimicrobial propensity only at the point of contact without spreading to a more cross-sectional area of application minimizing the risk of toxicity through excess release. This is also clearly evident from the small halo area in the zone of inhibition as depicted in Figure 5A.

HG-EGCG showed a preferential antibacterial activity towards the gram-negative bacteria compared to grampositive, ${ }^{13}$ which might be due to the presence of a thick 
A

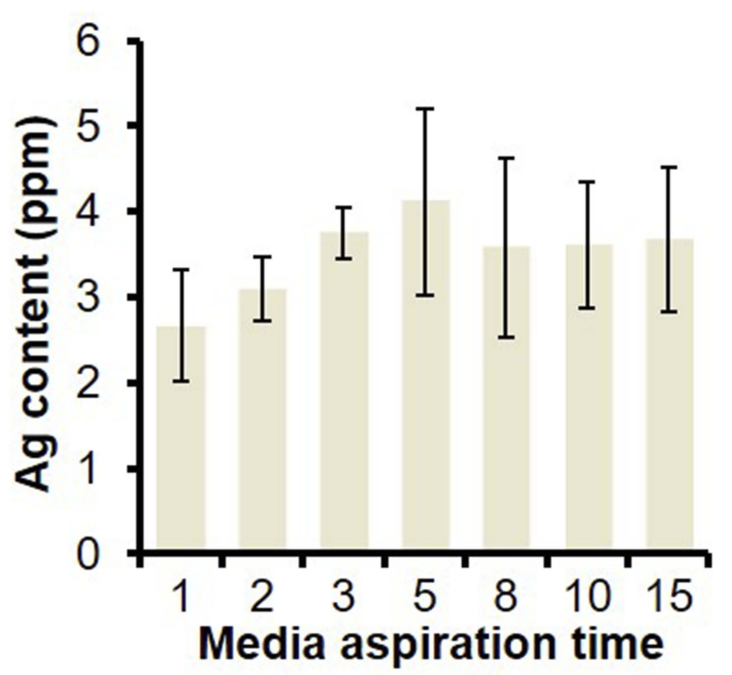

C

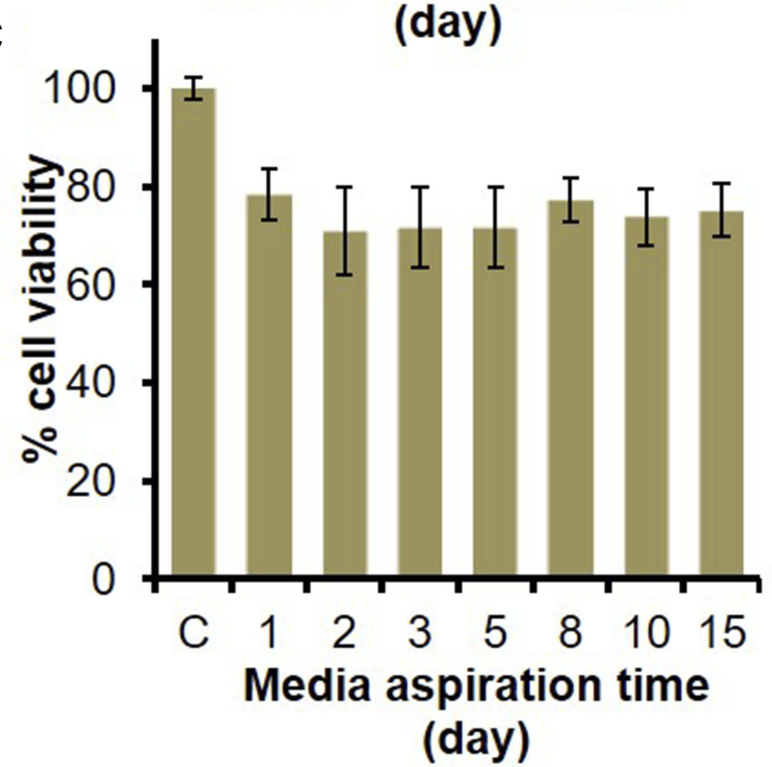

B
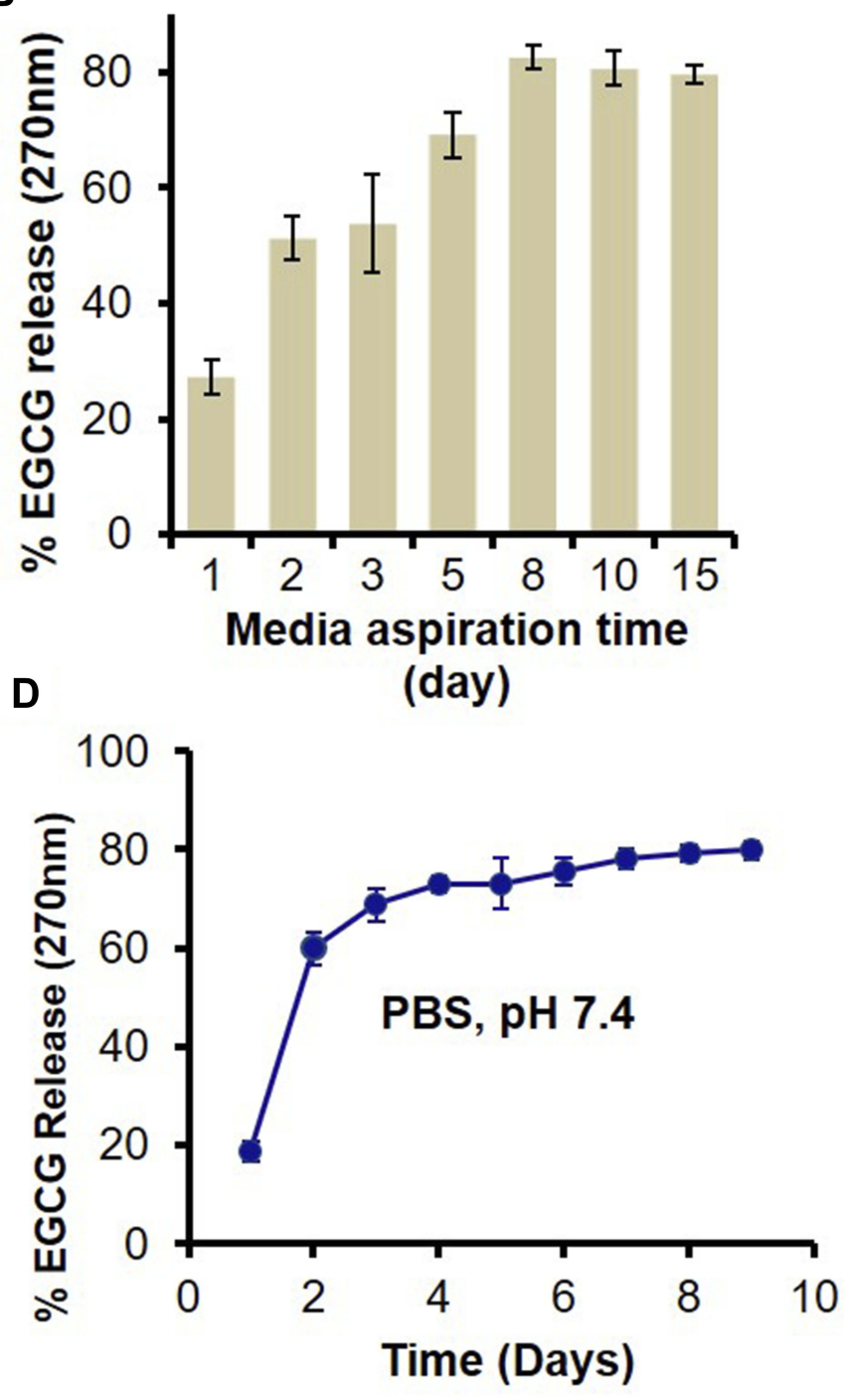

Figure $4 \mathrm{In}$ vitro release of $\mathrm{Ag}$ and EGCG from HG-Ag-EGCG and their effect on biocompatibility. HG-Ag-EGCG was placed in DMEM culture medium to unloose its content. The media was aspirated at different time points (I, 3, 5, 8, I0, I5 days) and subjected to analyses. (A) AAS estimation of Ag content with a maximum concentration of $4.1 \mathrm{Ppm}$. (B) UV analysis for EGCG release shows sustained release profile in aspirated media and $\sim 80 \%$ of EGCG was released in $8^{\text {th }}$ day which remains constant afterward. (C) MTT assay for cell viability on aspirated media treated normal skin MSC P5 cells indicates good biocompatibility of $\mathrm{Hg}$-Ag-EGCG. (D) EGCG release kinetics from final formulation ( $\mathrm{Hg}$-Ag-EGCG) at a different time interval in PBS; $\mathrm{pH} 7.4$ (mean $\pm \mathrm{SD}, \mathrm{n}=3$ ).

layer of peptidoglycans in the cell wall of S. aureus. ${ }^{51}$ Further, growth curve analysis was initiated against $B$. subtilis and E. coli, and the result affirmed that HG-Ag and HG-AgEGCG were quite lethal against both the strains (Figure 5B). Numerous earlier reports have established the antibacterial activity of $\mathrm{Ag}$, and more recently, Dakal et al have reviewed the mechanistic basis of Ag NPs involved. ${ }^{16,52}$ Different concentration of EGCG was also applied to check the antibacterial efficacy of EGCG, and it was found that EGCG shows bacteriostatic propensity in our case.

\section{HG-Ag-EGCG Boost Procoagulation}

\section{Leading to Rapid Wound Closure}

We next evaluated wound contraction and as shown in Figure 6A, brisk wound closure was noticed with HG-AgEGCG treated wounds compared to other treated groups at different days $(0,9$ th, 12 th and 15 th day). This difference was most conspicuous at day 9, wherein HG-Ag-EGCG treated group showed rapid wound closure under the dried scab with the smallest wound area ( $\sim 30 \%$ remaining to heal). Interestingly, our formulation elicits a fast response 


\section{Zone Inhibition Analysis}

A

E. coli (K12)
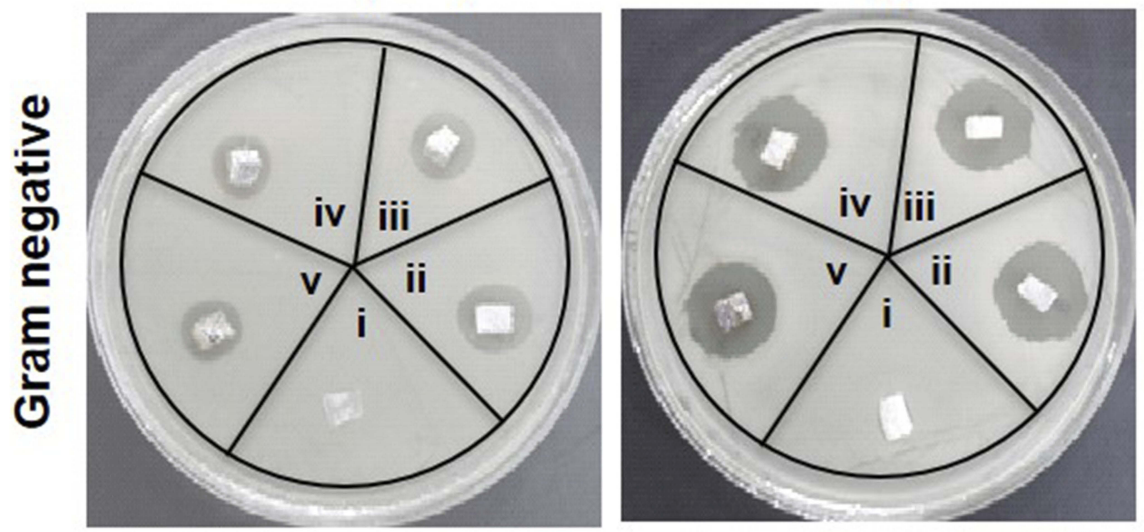

B. subtilis

S. aureus
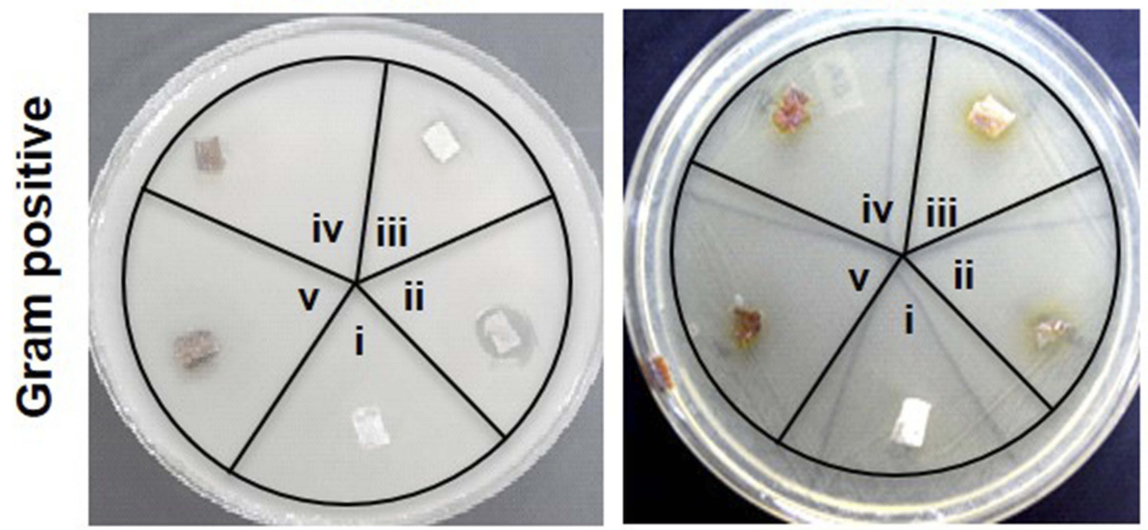

B
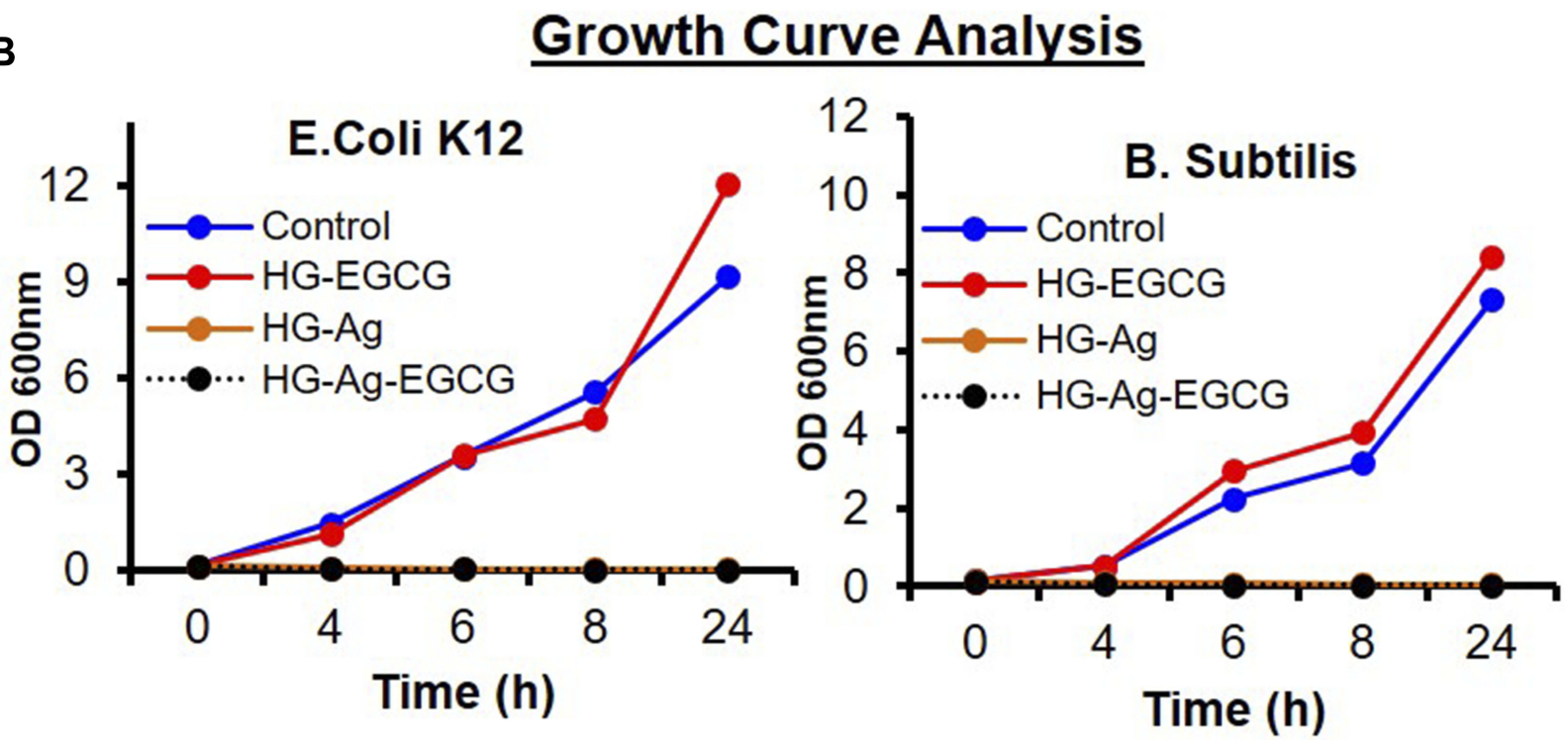

Figure 5 Antibacterial potency of different hydrogel patches. Digital image showing the antibacterial activity of different hydrogel patches against gram-negative (E. coli. and P. aeruginosa) and gram-positive (B. subtilis and S. Aureus) bacteria. (A) Zone inhibition assay ( $\mathrm{i}=\mathrm{HG}, \mathrm{ii}=\mathrm{HG}-\mathrm{Ag}$, iii = HG-EGCG, iv = HG-Ag-EGCG (I0 Ppm), v = HG-AgEGCG $(50 \mathrm{ppm})$ treatment time $12 \mathrm{hrs}$ ). (B) Growth curve analysis (treatment time $24 \mathrm{hrs}$ ) for $E$. coli. and B. subtilis. Antibacterial activity data clearly indicate a potent antibacterial property of $\mathrm{Hg}$-Ag-EGCG. 
A

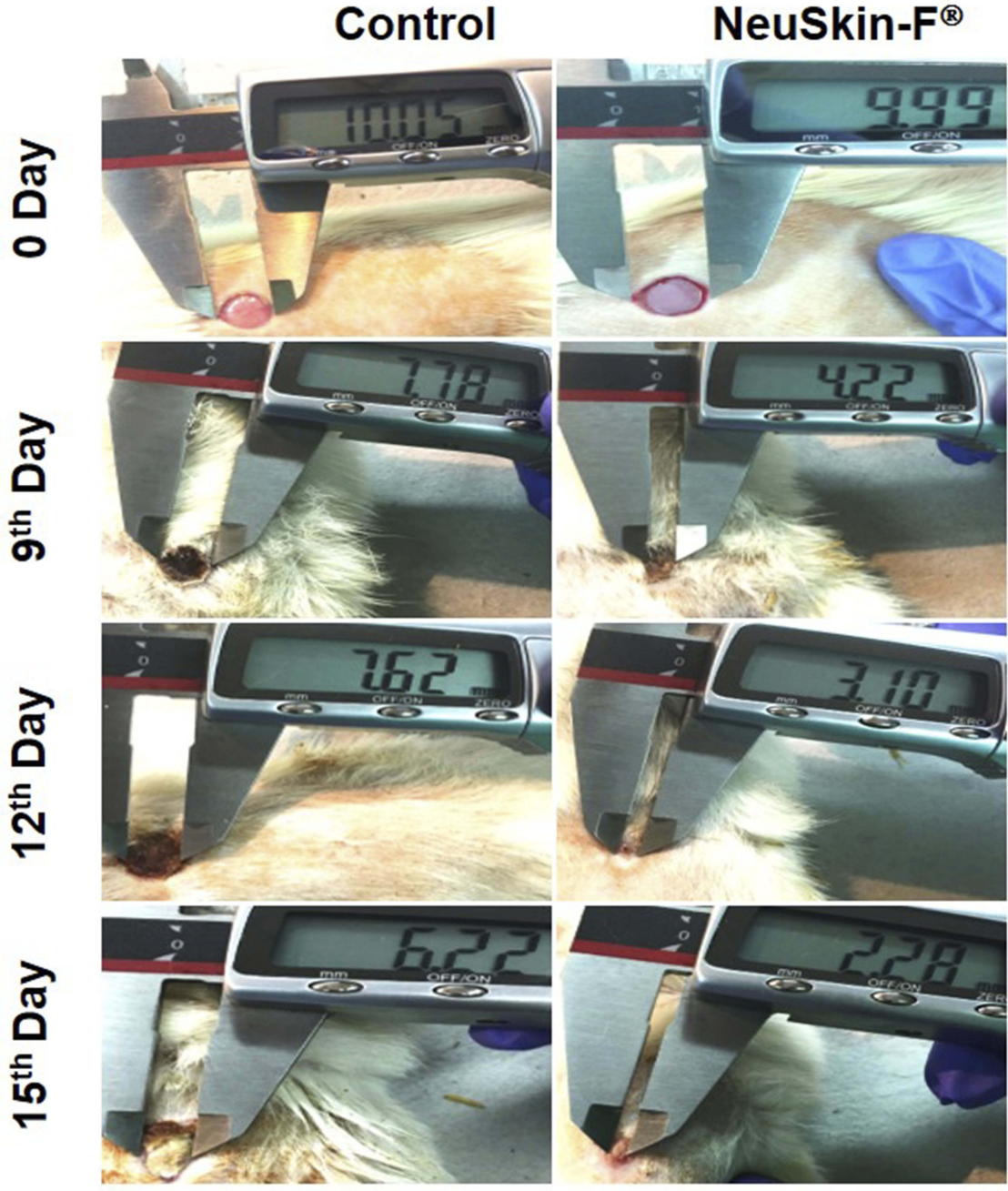

NeuSkin-F${ }^{\circledR}$

HG-Ag-EGCG
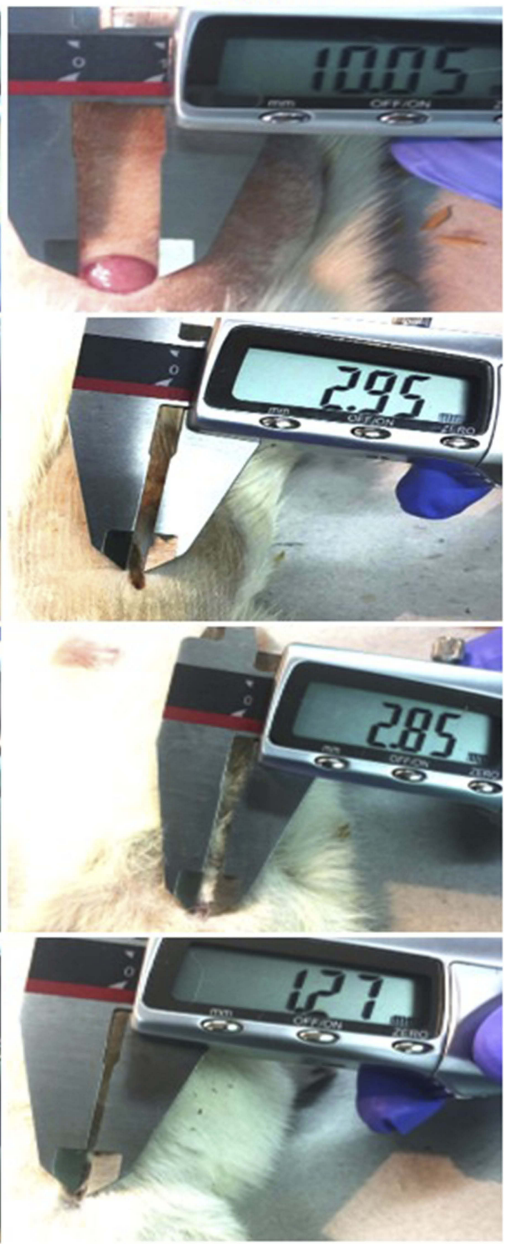

B

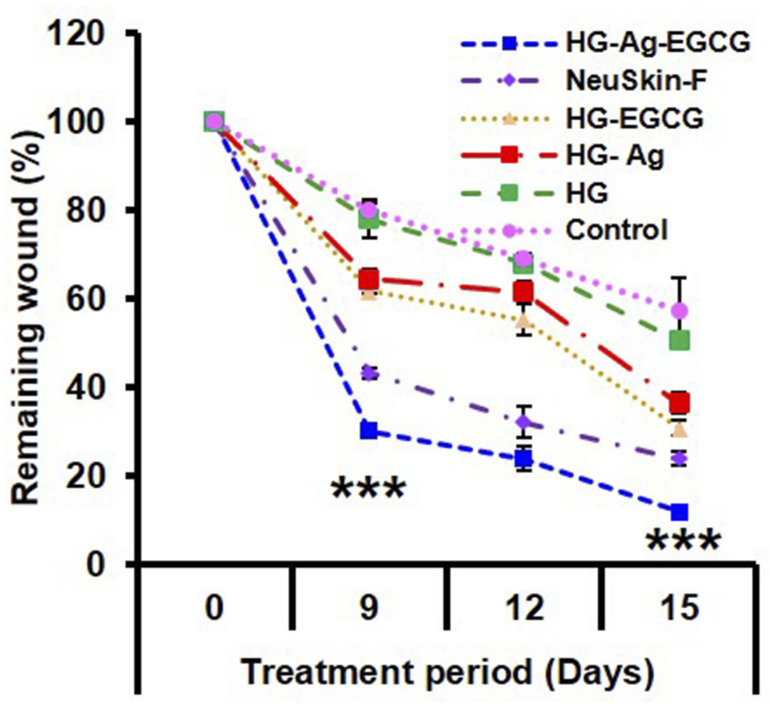

C Control

HG

HG-Agonly EGCG

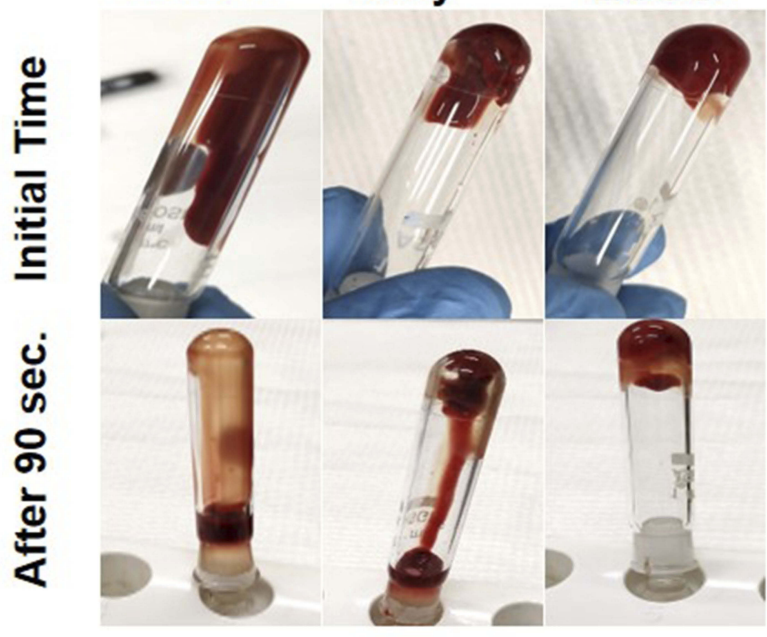

Figure 6 HG-Ag-EGCG's procoagulation and in vivo wound-healing potential. (A) Digitals images of wounds post-treatment with Hg-Ag-EGCG and NeuSkin- ${ }^{\circledR}$ compared to the negative control (bare wound). (B) Quantitative analysis of wound closure area on days $0,9,12$ and $15^{\text {th }}$ day post-treatment with different hydrogel nanocomposites (mean $\pm \mathrm{SD}, \mathrm{n}=3, * * * \mathrm{p}<0.000 \mathrm{I}$ ). It is to be noteworthy that the extent of wound closure in post-treatment with HG-Ag-EGCG hydrogel patch is superior compared to the commercially available wound patch (Images for confocal study were obtained at 20x magnification). (C) Blood cell coagulation effect of various wound patches. 
far better than control and commercially available, NeuSkin- $\mathrm{F}^{\circledR}$ treated groups which showed $\sim 75 \%$ and $45 \%$ ( $p<0.0001)$ remaining wound area, respectively (Figure 6B). Further, HG-EGCG showed only $6 \%$ lag in wound closure percentage on day 15 compared to the commercial film dressing NeuSkin- ${ }^{\circledR}$. Nonetheless, on day 15 , unlike control group wounds which showed $>$ $55 \%$ remaining wound area, HG-Ag-EGCG presented complete wound closure and higher resemblance with the normal skin with complete hair growth on and around wound surface. Above data demonstrated HG-Ag-EGCG promoted better wound healing with proper tissue architecture compared to other groups. We believe, antioxidant and antimicrobial properties of EGCG and Ag NPs, synergistically accelerating the healing ability as time passes. It has been reported that protein content around the wound area influences the Ag release, ${ }^{53}$ but its contact-dependent antibacterial activity aids additive effect in wound healing.

Coagulation time is an important and essential indicator of procoagulation potency of any hemostatic dressing materials. To confirm whether Hg-Ag-EGCG is inducing blood cell coagulation in heparinized mouse whole blood was used. It was found that HG-Ag-EGCG promotes this clinically relevant outcome more profoundly compared to HG alone. The blood remained free-flowing in $\mathrm{HG}$ even after $90 \mathrm{~s}$, while liquid blood was instantly (within a few seconds) transformed into a self-supporting gel with HGAg-EGCG where the sample held its weight even upon tube inversion (Figure 6C). The negative surface potential of the patches might be activating the platelets subsequently the platelet glycoprotein (GPIIb/IIIa) receptor becomes competent to bind soluble fibrinogen which bridges GPIIb/IIIa between adjacent platelets stimulating the release of various clotting. ${ }^{54}$

\section{HG-Ag-EGCG Potentiate Cell}

\section{Proliferation by Inducing KI67 Expression}

KI67 is a labile, non-histone nuclear protein that is tightly linked to the cell cycle and does not appear to be expressed during DNA repair processes and therefore, regarded as a marker of cell proliferation. In order to increase the wound-healing rate, it is important to impulse endogenous cells to promote skin cells proliferation. ${ }^{55}$ Moreover, all the active components of HG-Ag-EGCG are excellent promoter of cell proliferation and are carefully chosen. The immuno-histochemical staining with its quantification shows that HG-Ag-EGCG wound patch enhances the expression of cell proliferation marker KI67 up to six fold compared to control, indicating our hydrogel patch possesses excellent wound-healing ability as shown in Figure 7A and B.

\section{HG-Ag-EGCG Promotes Collagen Synthesis, Granulation, Neovascularization, and Epithelial Recovery}

The epidermal recovery is one of the key hallmarks of tissue regeneration and implicated in wound healing. By 12th day HG-Ag-EGCG treated group showed significant healing response and dense mature collagen deposition with clear well-formed and differentiated epithelium compared to the control and NeuSkin- $\mathrm{F}^{\circledR}$ groups (Figure 8A). The control group showed white-colored void spaces in the image indicating a lack of collagen content and its reticular arrangement and hence, a loose crust.

Other groups, HG, HG-EGCG and HG-Ag, displayed somewhat incomplete epithelium migration over the dermis, granulation tissue formation still under progress for remodeling of tissue architecture, suggests that our final formulation has synergistically elicited a profound impact on wound healing than each active components viz. Ag or EGCG acting alone. HG-Ag-EGCG induces complete reepithelialization with apparent granulation tissue formation, highest fibroblast density as observed in the H\&E sections studied. Qualitatively, densely packed keratinocytes, as well as new blood vessels formation, are also present (yellow arrows in Figure 8B) suggesting the healed wound has a higher degree of resemblance to the normal skin with better cosmesis.

\section{HG-Ag-EGCG plays a Role in Cytokine Modulation and VEGF Induction}

Wound healing involves a cascade of tightly regulated biological events involving inflammation, proliferation and remodeling phases which often overlaps with each other. Although cytokines are vital for various cell signaling events during the repair process, yet sometimes they act like a double-edged sword for their role in impaired wound healing. We next studied the expressions of cytokines IL-10, IL-6, IFN- $\gamma$, TNF- $\alpha$, and growth factor VEGF using multiplex assay. The levels of IFN- $\gamma$ in the HG-Ag-EGCG-treated group remains statistically insignificant during the healing period, indicating an ideal condition for rapid wound healing (Figure 9A). ${ }^{56}$ The role of IFN- $\gamma$ is already implicated in impaired wound 
A
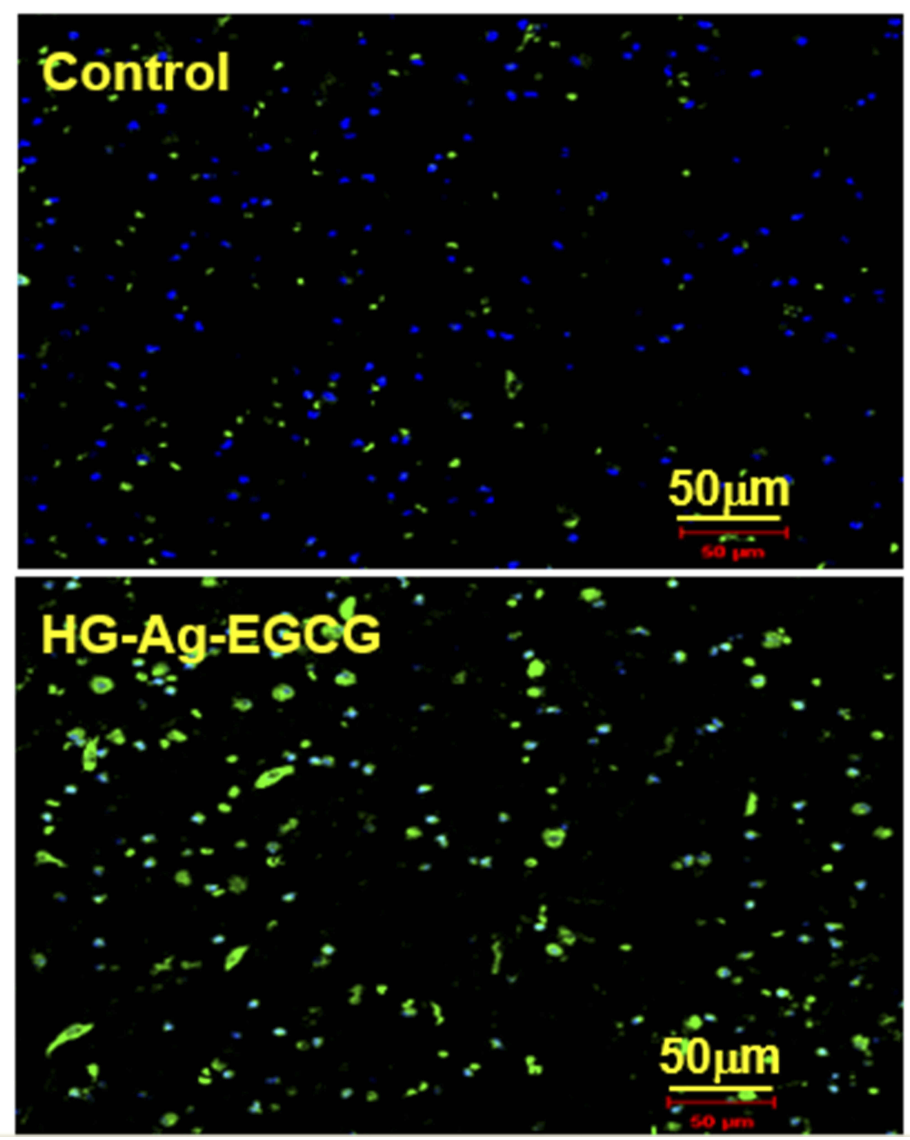

B

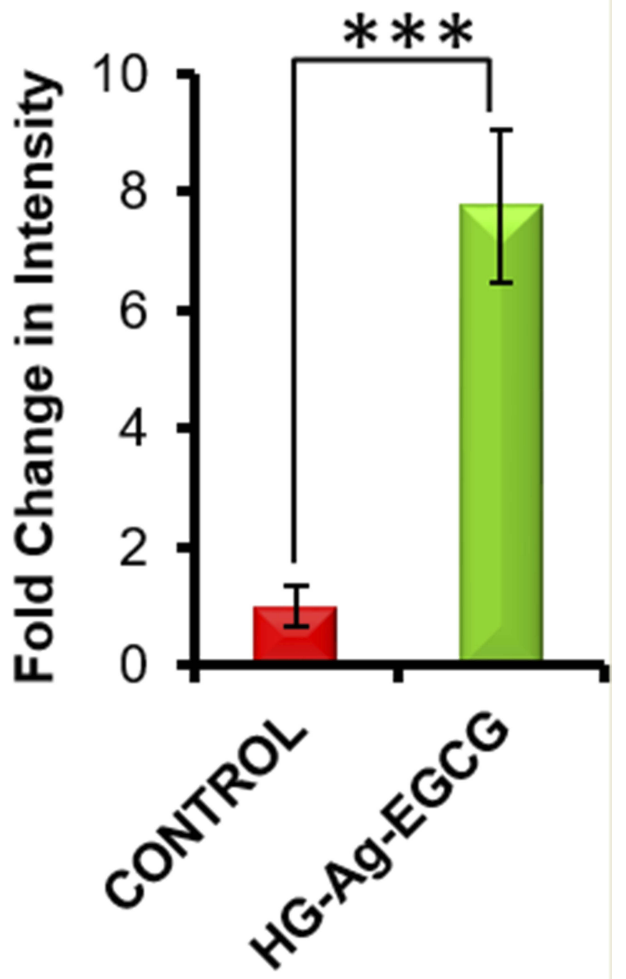

Figure 7 Cell proliferation ability of HG-Ag-EGCG. (A) Enhanced expression of KI 67 on hydrogel treated tissue sample (post 9-days treatment), examined by Immunohistochemistry under confocal microscopy. (B) Quantification data of the confocal image $(\mathrm{n}=3$, $* * * \mathrm{p}<0.0005)$. It is to be noteworthy that the extent of wound closure in post-treatment with HG-Ag-EGCG hydrogel wound patch is superior compared to the control group wound patch (Images of confocal study were obtained at $20 x$ magnification).

healing. ${ }^{57}$ The above finding prompts us to check expression levels of VEGF and we found the levels were upregulated till day 9 in HG-Ag-EGCG-treated groups compared to other groups, including commercial film dressing, NeuSkin- $\mathrm{F}^{\circledR}$ as presented in Figure 9E. The levels of pro-inflammatory cytokine, IL-6, decreased gradually during the whole healing process across all the formulations and are statistically significant with the final formulation $\mathrm{HG}-\mathrm{Ag}-\mathrm{EGCG}$, eliciting lowest level as shown in Figure 9B. However, the levels of anti-inflammatory IL-10 decreased significantly across the group and more prominently HG-Ag-EGCG group on day 9 showing its ant-inflammatory potential (Figure 9C). As is known, initial inflammation at the wound site is essential to initiate the repair process which needs to be brought down to its basal level which is manifested in levels of IL-10. Interestingly, the levels of TNF- $\alpha$ remain more or less unal- tered (Figure 9D) and may be the reason for facilitating accelerated wound healing. Nonetheless, the relative increment in the VEGF expression decreased with time in HG-AgEGCG treated group post 9th day presumably because the wound had already healed. At an early stage of wound healing, IL-6 and IL-10 are responsible for the recruitment of fibroblast as well as the removal of extracellular matrix debris, ${ }^{58}$ but, the prolonged presence of these cytokines delays wound-healing cascade by creating the perfect environment for bacterial infiltration and proliferation. ${ }^{59}$ Control group showed increased secretion of pro-inflammatory cytokine IL-10 and IL-6 till 9th and 15th day which might be due to increased exudates formation and its poor management whereas, HG-EGCG and HG-Ag-EGCG wound patches were showing a similar pattern which finally resulted in quick wound healing. 


\section{Masson trichrome-staining Hematoxylin and eosin (H\&E) staining}

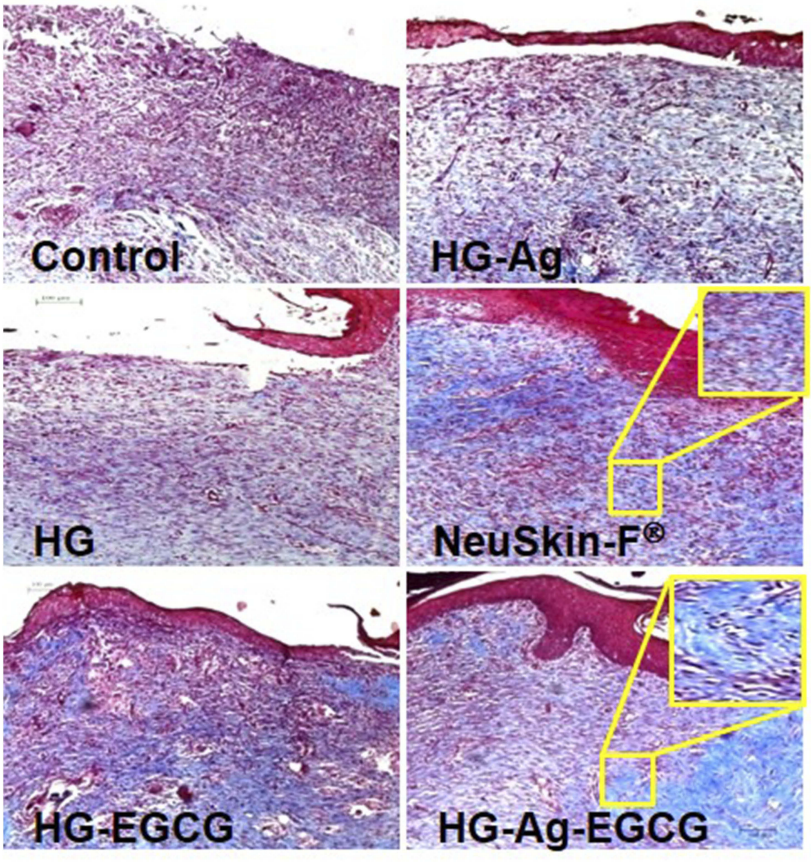

A

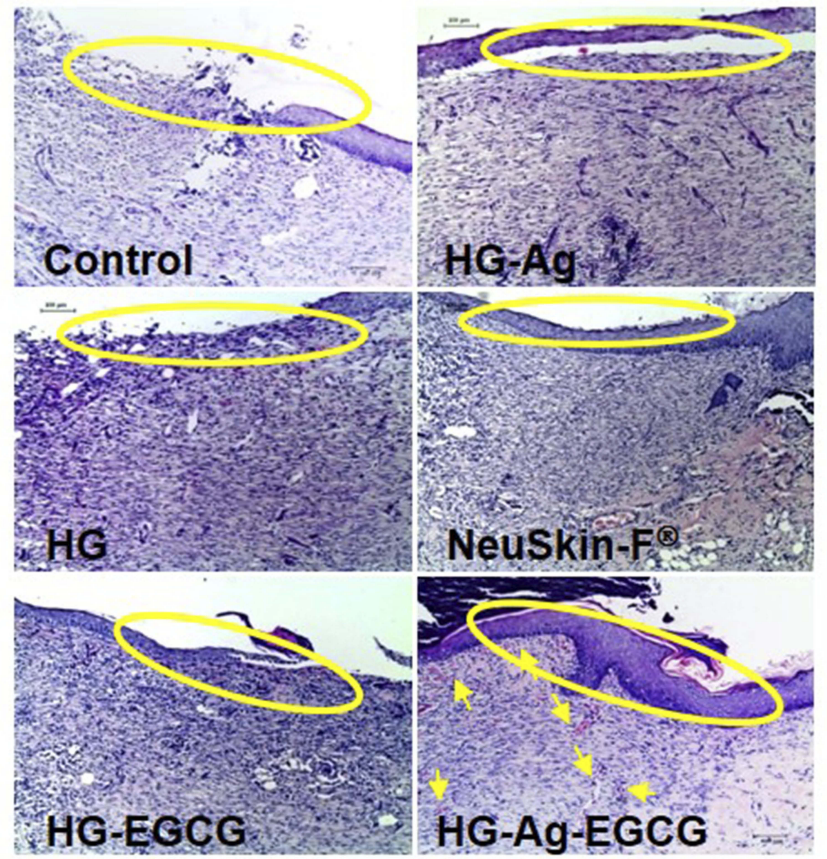

B

Figure 8 Effect of various hydrogel formulations on collagen synthesis and re-epithelialization. 12 days treated skin samples around the wound area were taken, and $5 \mu$ thin samples were cut by microtome for histo-pathological examination. (A) Representative photomicrographs of Masson trichrome-stained skin section showing enhanced collagen synthesis, firm-differentiated epithelium, and no visible inflammation or infection on HG-Ag-EGCG-treated sections while the control group showed more void spaces with loose crust. (B) Hematoxylin and eosin (H\&E) stain shows HG-Ag-EGCG-exposed group has successful reepithelialization, granular tissue formation with enhanced neovascularization and densely packed keratinocytes (yellow arrows) $(\mathrm{n}=3$, scale bar $100 \mu \mathrm{m})$.

\section{HG-Ag-EGCG Is Non-Toxic to Kidney and Liver Function}

We further assessed the systemic toxicity by measuring the serum level of AST, ALT for liver function and Urea, Creatinine for kidney function. As anticipated, histological examination of liver and kidney confirmed no histo-architecture alteration post-HG-Ag-EGCG treatment suggesting that our hydrogel offers a unique advantage in treating the subcutaneous wound in a better way (Figure S4A). Further, no significant alteration in ALT, AST, urea, and creatinine was observed between control and HG-Ag-EGCG treated groups post wounding till day 15 (Figure S4B).

\section{Conclusion}

The present work focuses on facile green synthesis of Ag NPs embedded in natural polymer-based hydrogels patch towards more efficient and accelerated wound healing. The optimum content of all the active components, gelatin, alginate, Ag NPs, and EGCG in the final formulation imparts antibacterial and anti-inflammatory responses. The wound patch displayed good cytocompatibility towards normal keratinocytes MSC P5 cells. The formulation was endowed with monodispersed $\mathrm{Ag}$ NPs and withstood sustained release of EGCG from the matrix to the wound bed. Hg-Ag-EGCG showed significant swelling, porosity and tensile strength providing an apt moist environment for a smooth transition between inflammatory-proliferation-remodeling phases. Toxicity study also revealed our formulation is non-toxic to kidney, liver and other blood parameters. Further, our formulation elicit favorable conditions for the different phases of wound healing and promotes rapid wound healing. Nonetheless, customization of our formulation to improve its tensile strength by further modification and optimization is possible for future applications in wound healing. Overall, this study as summarized in Figure S5 improves our understanding of natural polymer-based hydrogel patch for speedy wound healing by synergistically combining antioxidant and antibacterial properties. 

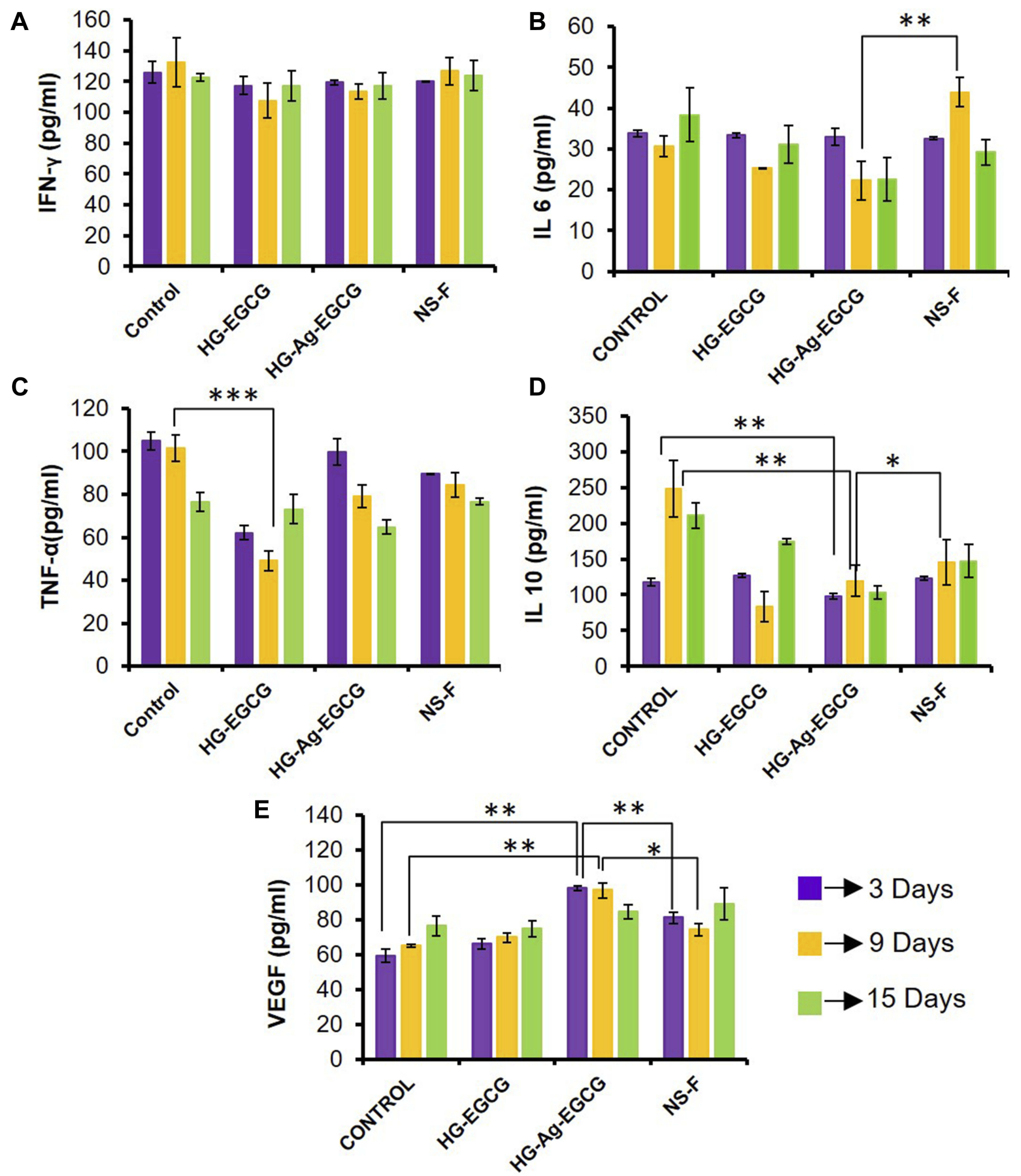

Figure 9 Effect of hydrogel patches on growth factor and cytokines. (A) Unaltered expression levels of IFN- $\gamma$ secretion. (B and C) Inhibition of pro-inflammatory cytokines IL-6 and TNF- $\alpha$ secretion on treatment with HG-Ag-EGCG. (D) Significant changes found in anti-inflammatory IL-I0 secretion on HG-Ag-EGCG treated group (E) Enhanced VEGF secretion on HG-Ag-EGCG treated group $\left(n=3, * * * p=0.0006, *^{*} p<0.005,{ }^{*} p<0.05\right)$.

\section{Acknowledgments}

A.K.K. and M.P.P. were supported by UGC-SRF fellowship. N.D. was supported by CSIR-SRF fellowship. A.S. was supported by ICMR-SRF fellowship. The authors also acknowledge Dr. V. Singh and Dr. R. Gera for their help in multiplexing and immunohistochemistry. We acknowledge 
Mr. K. Mandrah's valuable help in AAS examination and Miss Khyati from IIT, Mandi, India, for helping in tensile strength examination. The CSIR-IITR manuscript No. is 3589. Dr Mohan Kamthan is currently affiliated with the School of Chemical and Life Sciences, Department of Biochemistry, Jamia Hamdard, Hamdard Nagar, New Delhi-110062, Delhi, India.

\section{Author Contributions}

The manuscript was written through the contributions of all authors. All authors contributed to data analysis, drafting or revising the article, gave final approval of the version to be published, and agree to be accountable for all aspects of the work.

\section{Funding}

Partial financial support for this work from DBT and DST (GAP 368, 2018: GAP 375, 2019), Ministry of Science and Technology, is gratefully acknowledged.

\section{Disclosure}

The authors report no conflicts of interest in this work.

\section{References}

1. Boateng JS, Matthews KH, Stevens HNE, Eccleston GM. Wound healing dressings and drug delivery systems: a review. J Pharm Sci. 2008;97:2892-2923. doi:10.1002/jps.21210

2. Du L, Feng X, Xiang X, Jin Y. Wound healing effect of an in situ forming hydrogel loading curcumin-phospholipid complex. Curr Drug Deliv. 2016;13:76-82. doi:10.2174/1567201813666151202195437

3. Telgenhoff D, Shroot B. Cellular Senescence Mechanisms in Chronic Wound Healing. Nature Publishing Group; 2005.

4. Gopinath D, Ahmed MR, Gomathi K, Chitra K, Sehgal PK, Jayakumar R. Dermal wound healing processes with curcumin incorporated collagen films. Biomaterials. 2004;25:1911-1917. doi:10.1016/ S0142-9612(03)00625-2

5. Lai J-J, Lai K-P, Chuang K-H, et al. Monocyte/macrophage androgen receptor suppresses cutaneous wound healing in mice by enhancing local TNF- $\alpha$ expression. $J$ Clin Invest. 2009;119:3739-3751. doi:10.1172/JCI39335

6. Ueno H, Mori T, Fujinaga T. Topical formulations and wound healing applications of chitosan. Adv Drug Deliv Rev. 2001;52:105-115. doi:10.1016/S0169-409X(01)00189-2

7. Schreml S, Szeimies RM, Prantl L, Karrer S, Landthaler M, Babilas P. Oxygen in acute and chronic wound healing. $\mathrm{Br}$ $J$ Dermatol. 2010;163:257-268. doi:10.1111/bjd.2010.163.issue-2

8. Panchatcharam M, Miriyala S, Gayathri VS, Suguna L. Curcumin improves wound healing by modulating collagen and decreasing reactive oxygen species. Mol Cell Biochem. 2006;290:87-96. doi:10.1007/s11010-006-9170-2

9. Leu J-G, Chen S-A, Chen H-M, et al. The effects of gold nanoparticles in wound healing with antioxidant epigallocatechin gallate and $\alpha$-lipoic acid. Nanomed. 2012;8:767-775. doi:10.1016/j.nano.2011.08.013

10. Mohanty C, Das M, Sahoo SK. Sustained wound healing activity of curcumin loaded oleic acid based polymeric bandage in a rat model. Mol Pharm. 2012;9:2801-2811. doi:10.1021/mp300075u
11. Lin YH, Lin JH, Li TS, et al. Dressing with epigallocatechin gallate nanoparticles for wound regeneration. Wound Repair Regener. 2015;24:287-301.

12. Kapoor M, Howard R, Hall I, Appleton I. Effects of epicatechin gallate on wound healing and scar formation in a full thickness incisional wound healing model in rats. Am $J$ Pathol. 2004;165:299-307. doi:10.1016/S0002-9440(10)63297-X

13. Nakayama M, Shimatani K, Ozawa $T$, et al. Mechanism for the antibacterial action of epigallocatechin gallate (EGCg) on Bacillus subtilis. Biosci Biotechnol Biochem. 2015;79:845-854. doi:10.1080/ 09168451.2014 .993356

14. Annabi N, Rana D, Shirzaei Sani E, et al. Engineering a sprayable and elastic hydrogel adhesive with antimicrobial properties for wound healing. Biomaterials. 2017;139:229-243. doi:10.1016/j. biomaterials.2017.05.011

15. Richter AP, Brown JS, Bharti B, et al. An environmentally benign antimicrobial nanoparticle based on a silver-infused lignin core. Nat Nanotechnol. 2015;10:817. doi:10.1038/nnano.2015.141

16. Dakal TC, Kumar A, Majumdar RS, Yadav V. Mechanistic basis of antimicrobial actions of silver nanoparticles. Front Microbiol. 2016;7:1831. doi:10.3389/fmicb.2016.01831

17. Panacek A, Kolar M, Vecerova R, et al. Antifungal activity of silver nanoparticles against Candida spp. Biomaterials. 2009;30:6333-6340. doi:10.1016/j.biomaterials.2009.07.065

18. Poole K, Krebes K, McNally C, Neshat S. Multiple antibiotic resistance in Pseudomonas aeruginosa: evidence for involvement of an efflux operon. $J$ Bacteriol. 1993;175:7363-7372. doi:10.1128/ jb.175.22.7363-7372.1993

19. Sondi I, Salopek-Sondi B. Silver nanoparticles as antimicrobial agent: a case study on E. coli as a model for gram-negative bacteria. J Colloid Interface Sci. 2004;275:177-182. doi:10.1016/j.jcis.2004.02.012

20. Taylor DL, In Het Panhuis M. Self-healing hydrogels. Adv Mater. 2016;28:9060-9093. doi:10.1002/adma.201601613

21. Zhang L, Ma Y, Pan X, Chen S, Zhuang H, Wang S. A composite hydrogel of chitosan/heparin/poly ( $\hat{\mathrm{I}}^{3}$-glutamic acid) loaded with superoxide dismutase for wound healing. Carbohydr Polym. 2018;180:168-174. doi:10.1016/j.carbpol.2017.10.036

22. Verma NK, Purohit MP, Equbal D, et al. Targeted smart $\mathrm{pH}$ and thermoresponsive N,O-carboxymethyl chitosan conjugated nanogels for enhanced therapeutic efficacy of doxorubicin in MCF-7 breast cancer cells. Bioconjug Chem. 2016;27:2605-2619. doi:10.1021/acs. bioconjchem.6b00366

23. Chen G, Yu Y, Wu X, Wang G, Ren J, Zhao Y. Bioinspired multifunctional hybrid hydrogel promotes wound healing. Adv Funct Mater. 2018;28:1801386. doi:10.1002/adfm.201801386

24. Chen W-Y, Chang H-Y, Lu J-K, et al. Self-assembly of antimicrobial peptides on gold nanodots: against multidrug-resistant bacteria and wound-healing application. Adv Funct Mater. 2015;25:7189-7199. doi:10.1002/adfm.201503248

25. Dong Y, Rodrigues M, Li X, et al. Injectable and tunable gelatin hydrogels enhance stem cell retention and improve cutaneous wound healing. Adv Funct Mater. 2017;27:1606619. doi:10.1002/adfm.201606619

26. Xiao J, Chen S, Yi J, Zhang HF, Ameer GA. A cooperative copper metal-organic framework-hydrogel system improves wound healing in diabetes. Adv Funct Mater. 2017;27:1604872. doi:10.1002/adfm.v27.1

27. Xie Z, Aphale NV, Kadapure TD, et al. Design of antimicrobial peptides conjugated biodegradable citric acid derived hydrogels for wound healing. J Biomed Mater Res Part A. 2015;103:3907-3918. doi:10.1002/jbm.a.35512

28. Balakrishnan B, Mohanty M, Umashankar P, Jayakrishnan A. Evaluation of an in situ forming hydrogel wound dressing based on oxidized alginate and gelatin. Biomaterials. 2005;26:6335-6342. doi:10.1016/j.biomaterials.2005.04.012

29. Qiu Y, Park K. Environment-sensitive hydrogels for drug delivery. Adv Drug Deliv Rev. 2001;53:321-339. doi:10.1016/S0169-409X(01) 00203-4 
30. Ghosh Auddy R, Abdullah MF, Das S, Roy P, Datta S, Mukherjee A. New guar biopolymer silver nanocomposites for wound healing applications. Biomed Res Int. 2013;2013.

31. Ishihara M, Nakanishi K, Ono K, et al. Photocrosslinkable chitosan as a dressing for wound occlusion and accelerator in healing process. Biomaterials. 2002;23:833-840. doi:10.1016/S0142-9612(01)00189-2

32. Obara $\mathrm{K}$, Ishihara $\mathrm{M}$, Ishizuka $\mathrm{T}$, et al. Photocrosslinkable chitosan hydrogel containing fibroblast growth factor-2 stimulates wound healing in healing-impaired db/db mice. Biomaterials. 2003;24:3437-3444. doi:10.1016/S0142-9612(03)00220-5

33. Purohit MP, Verma NK, Kar AK, Singh A, Ghosh D, Patnaik S. Inhibition of thioredoxin reductase by targeted selenopolymeric nanocarriers synergizes the therapeutic efficacy of doxorubicin in MCF7 human breast cancer cells. ACS Appl Mater Interfaces. 2017;9:36493-36512. doi:10.1021/acsami.7b07056

34. Mohandas A, Sudheesh Kumar P, Raja B, Lakshmanan V-K, Jayakumar R. Exploration of alginate hydrogel/nano zinc oxide composite bandages for infected wounds. Int $J$ Nanomedicine. 2015;10:53.

35. Balakrishnan B, Mohanty M, Umashankar PR, Jayakrishnan A. Evaluation of an in situ forming hydrogel wound dressing based on oxidized alginate and gelatin. Biomaterials. 2005;26:6335-6342. doi:10.1016/j.biomaterials.2005.04.012

36. Lee KY, Mooney DJ. Alginate: properties and biomedical applications. Prog Polym Sci. 2012;37:106-126. doi:10.1016/j. progpolymsci.2011.06.003

37. Gilchrist T, Martin AM. Wound treatment with Sorbsan-an alginate fibre dressing. Biomaterials. 1983;4:317-320. doi:10.1016/01429612(83)90036-4

38. Truong VX, Hun ML, Li F, Chidgey AP, Forsythe JS. In situ-forming click-crosslinked gelatin based hydrogels for 3D culture of thymic epithelial cells. Biomater Sci. 2016;4:1123-1131. doi:10.1039/ C6BM00254D

39. Draye JP, Delaey B, Van de Voorde A, Van Den Bulcke A, Bogdanov B, Schacht E. In vitro release characteristics of bioactive molecules from dextran dialdehyde cross-linked gelatin hydrogel films. Biomaterials. 1998;19:99-107. doi:10.1016/S0142-9612(97) 00164-6

40. Chong E, Phan T, Lim I, et al. Evaluation of electrospun PCL/gelatin nanofibrous scaffold for wound healing and layered dermal reconstitution. Acta Biomater. 2007;3:321-330. doi:10.1016/j.actbio.2007.01.002

41. Stout EI, McKessor A. Glycerin-based hydrogel for infection control. Adv Wound Care. 2012;1:48-51. doi:10.1089/wound.2011.0288

42. Murali R, Vidhya P, Thanikaivelan P. Thermoresponsive magnetic nanoparticle-aminated guar gum hydrogel system for sustained release of doxorubicin hydrochloride. Carbohydr Polym. 2014;110:440-445. doi:10.1016/j.carbpol.2014.04.076

43. Sudheesh Kumar PT, Lakshmanan V-K, Anilkumar TV, et al. Flexible and microporous chitosan hydrogel/nano $\mathrm{ZnO}$ composite bandages for wound dressing: in vitro and in vivo evaluation. $A C S$ Appl Mater Interfaces. 2012;4:2618-2629. doi:10.1021/am300292v

44. Moreno-Bautista G, Tam KC. Evaluation of dialysis membrane process for quantifying the in vitro drug-release from colloidal drug carriers. Colloids Surf A. 2011;389:299-303. doi:10.1016/j.colsurfa.2011.07.032
45. Dhiman N, Singh A, Verma NK, Ajaria N, Patnaik S. Statistical optimization and artificial neural network modeling for acridine orange dye degradation using in-situ synthesized polymer capped ZnO nanoparticles. J Colloid Interface Sci. 2017;493:295-306. doi:10.1016/j.jcis.2017.01.042

46. Zhao F, Yao D, Guo R, Deng L, Dong A, Zhang J. Composites of polymer hydrogels and nanoparticulate systems for biomedical and pharmaceutical applications. Nanomaterials. 2015;5:2054-2130. doi:10.3390/nano5042054

47. Latifi N, Asgari M, Vali H, Mongeau L. A tissue-mimetic nano-fibrillar hybrid injectable hydrogel for potential soft tissue engineering applications. Sci Rep. 2018;8:1047. doi:10.1038/s41598-017-18523-3

48. Han L, Yan L, Wang K, et al. Tough, self-healable and tissue-adhesive hydrogel with tunable multifunctionality. Npg Asia Mater. 2017;9:e372. doi:10.1038/am.2017.33

49. Chen H, Cheng R, Zhao X, et al. An injectable self-healing coordinative hydrogel with antibacterial and angiogenic properties for diabetic skin wound repair. NPG Asia Mater. 2019;11:3. doi:10.1038/ s41427-018-0103-9

50. Johnston HJ, Hutchison G, Christensen FM, Peters S, Hankin S, Stone V. A review of the in vivo and in vitro toxicity of silver and gold particulates: particle attributes and biological mechanisms responsible for the observed toxicity. Crit Rev Toxicol. 2010;40:328-346. doi:10.3109/10408440903453074

51. Shahverdi AR, Fakhimi A, Shahverdi HR, Minaian S. Synthesis and effect of silver nanoparticles on the antibacterial activity of different antibiotics against Staphylococcus aureus and Escherichia coli. Nanomed. 2007;3:168-171. doi:10.1016/j.nano.2007.02.001

52. Xiu Z-M, Zhang Q-B, Puppala HL, Colvin VL, Alvarez PJJ. Negligible particle-specific antibacterial activity of silver nanoparticles. Nano Lett. 2012;12:4271-4275. doi:10.1021/n1301934w

53. Shao J, Wang B, Li J, Jansen JA, Walboomers XF, Yang F. Antibacterial effect and wound healing ability of silver nanoparticles incorporation into chitosan-based nanofibrous membranes. Mater Sci Eng. 2019;98:1053-1063. doi:10.1016/j.msec.2019.01.073

54. Fullard JF. The role of the platelet glycoprotein IIb/IIIa in thrombosis and haemostasis. Curr Pharm Des. 2004;10:1567-1576. doi:10.2174/ 1381612043384682

55. Mao C, Xiang Y, Liu X, et al. Repeatable photodynamic therapy with triggered signaling pathways of fibroblast cell proliferation and differentiation to promote bacteria-accompanied wound healing. $A C S$ Nano. 2018;12:1747-1759. doi:10.1021/acsnano.7b08500

56. Ishida Y, Kondo T, Takayasu T, Iwakura Y, Mukaida N. The essential involvement of cross-talk between IFN-gamma and TGF-beta in the skin wound-healing process. J Immunol. 2004;172:1848-1855. doi:10.4049/jimmunol.172.3.1848

57. Miles RH, Paxton TP, Zacheis D, Dries DJ, Gamelli RL. Systemic administration of interferon-gamma impairs wound healing. $J$ Surg Res. 1994;56:288-294. doi:10.1006/jsre.1994.1045

58. Chen R, Wang J, Liu C. Biomaterials act as enhancers of growth factors in bone regeneration. Adv Funct Mater. 2016;26:8810-8823. doi:10.1002/adfm.201603197

59. Gethin G. Understanding the inflammatory process in wound healing. Br J Community Nurs. 2012;17:S17-S22. doi:10.12968/bjen.2012.17. Sup3.S17
International Journal of Nanomedicine

\section{Publish your work in this journal}

The International Journal of Nanomedicine is an international, peerreviewed journal focusing on the application of nanotechnology in diagnostics, therapeutics, and drug delivery systems throughout the biomedical field. This journal is indexed on PubMed Central, MedLine, CAS, SciSearch ${ }^{\mathbb{R}}$, Current Contents ${ }^{\mathbb{R}} /$ Clinical Medicine, $^{2}$
Journal Citation Reports/Science Edition, EMBase, Scopus and the Elsevier Bibliographic databases. The manuscript management system is completely online and includes a very quick and fair peer-review system, which is all easy to use. Visit http://www.dovepress.com/ testimonials.php to read real quotes from published authors. 